\title{
Varieties of mobility measures: Comparing survey and mobile phone data during the COVID-19 pandemic
}

\author{
Fabian Kalleitner ${ }^{*}$, , David W. Schiestl ${ }^{2}$, and Georg Heiler ${ }^{3}$ \\ * corresponding author \\ ${ }^{1}$ University of Vienna, Department of Economic Sociology, Kolingasse 14-16, Vienna, 1090, Austria. \\ (fabian.kalleitner@univie.ac.at) \\ ${ }^{2}$ University of Vienna, Department of Economic Sociology, Kolingasse 14-16, Vienna, 1090, Austria. \\ (david.wolfgang.schiestl@univie.ac.at) \\ ${ }^{3}$ Complexity Science Hub Vienna, Josefstädter Straße 39, Vienna, 1080, Austria \& TU Wien, \\ Institute of Information Systems Engineering, Favoritenstraße 9-11/194, Vienna, 1040, Austria. \\ (heiler@csh.ac.at)
}

February 02, 2022

\begin{abstract}
Human mobility has become a major variable of interest during the COVID-19 pandemic and central to policy decisions all around the world. To measure individual mobility, research relies on a variety of indicators that commonly stem from two main data sources: survey-selfreports and behavioral mobility data from mobile phones. However, little is known about how mobility from survey self-reports relates to popular mobility estimates using GSM and GPS data. Spanning March 2020 until April 2021, this study compares self-reported mobility from a panel survey in Austria to aggregated mobility estimates utilizing (i) GSM data and (ii) Google's GPS-based Community Mobility Reports. Our analyses show that correlations in mobility changes over time are high, both in general and when comparing subgroups by age, gender, and mobility category. However, while these trends are similar, the size of relative mobility changes over time differs substantially between different mobility estimates. Overall, while our findings suggest that these mobility estimates manage to capture similar latent variables, especially when focusing on changes in mobility over time, researchers should be aware of the specific form of mobility different data sources capture.
\end{abstract}




\section{Introduction}

Since the start of the COVID-19 pandemic, social scientific research has contributed immensely to the understanding of human behavior in times of a global health crisis (Bavel et al. 2020). One crucial aspect in this endeavour is the analysis of human mobility (Haug et al. 2020; $\mathrm{Chu}$ et al. 2020). Empirical studies analyzing the consequences of non-pharmaceutical interventions such as school closings or retail closings have been used extensively to inform governments about the effectiveness of these policies (Alfano and Ercolano 2020; Schlosser et al. 2020; Vinceti et al. 2020). At the same time, researchers began to investigate the micro-level mechanisms explaining mobility changes to enable governments to tailor measures more effectively (Pfattheicher et al. 2020; Heffner, Vives, and FeldmanHall 2021, among many others).

These research goals produced two rather separate strands in the literature in which scholars typically rely on one of two distinct approaches to measure mobility. First, social scientists interested in micro-level mechanisms mostly rely on surveys asking individuals to self-report their mobility. This individual-level data allows researchers to study important covariates or implement experimental manipulations aiming to uncover the social mechanisms that explain the (un)effectiveness of certain policy changes (Kittel, Kalleitner, and Schiestl 2021; Jordan, Yoeli, and Rand 2020). However, using self-reports to estimate individuals' mobility also entails several drawbacks: next to the high expenses of surveys, self-reports include the risk of measurement errors due to biases and are limited in their measurement frequency.

Second, new technological innovations enabled researchers to use mobility data from mobile phones on a large scale to trace people's actual behaviors. These data were obtained either directly from service providers or indirectly via third-party sources and make use of data from user's device sensors (such as GPS) or from the mobile phone network (e.g. based on Global System for Mobile (GSM) communications) (Grantz et al. 2020; Buckee et al. 2020; Oliver et al. 2020). Mobility estimates from mobile phones became the dominant sources for calibrating epidemiological models (Vollmer et al. 2020), and for testing the effectiveness of governmental measures (Jay et al. 2020; Pepe et al. 2020). However, while these data are cost-effective and carry the potential to cover large areas (Zhang et al. 2021), they often lack information on potential explanatory variables of mobility and face potential coverage issues.

Despite differential advantages, so far, only a limited number of studies combine "sensor data" and survey data (Gollwitzer et al. 2020; Kitamura and Yamada 2020). This might partially be explained by issues like data protection, data availability, and limited experience with these new methods in social survey research (Struminskaya et al. 2021). For these reasons, two rather distinct fields of COVID-19 mobility research emerged, either using mobile phone data or using survey data. This opens a gap in the literature as we do not know whether commonly used mobility measures using survey self-reports behave similarly to those using behavioral data from mobile phones.

Several potential sources of representation and measurement errors could produce differences in mobility estimates depending on the data source used: User's limited ability to selfreport their mobility behavior in surveys renders detailed questions on the travelled distances 
impossible and may introduce social desirability bias and recall bias (Schwarz 2007). Contrary to that, data based on device sensors (such as GPS) offer high positioning accuracy and measurement frequency but may face coverage errors because of the limited number and distribution of users willing to participate in passive mobile data collection (Keusch et al. 2019; Rojas, Sadeghvaziri, and Jin 2016). Compared to data from device sensors, data based on GSM communications commonly comprise a larger number of users but are less exact in positioning (Gauvin et al. 2020).

The present article compares mobility trajectories across these three data sources using datasets that capture mobility patterns in Austria over a long period: Google's (GPS-based) Community Mobility Reports, aggregated GSM mobility data from a large Austrian internet service provider, and self-reported mobility from a representative web-based panel survey.

\section{Data and Methods}

For our survey estimates, we use data from a non probability web-survey panel provided by the Austrian Corona Panel Project (ACPP) (Kittel et al.2020a; 2020b). The panel consists of approximately 1500 participants per wave in 22 waves, spanning March 2020 until April of 2021. Survey respondents were recruited from a pre-existing commercial online access panel. Respondents needed to reside in Austria, be at least 14 years old, and have access to the internet. Respondents were invited based on quota sampling. Survey waves were fielded on a weekly (wave 1-10), bi-weekly (wave 11-14), and monthly basis (wave 15-22). To measure mobility, we calculate an additive index summarizing the answers to questions about the frequency respondents left their homes in the preceding 7 days for the following reasons: work, sports, meeting friends or relatives, buying medicine or receiving medical treatments, buying food, buying non-food products, walking pets, boredom, and other reasons (respondents could answer on a 5-point rating scale ranging from never to daily). Because few respondents are older than 74 (3.03\%), we limit our sample to those younger than 75 . Applying listwise deletion of missing values, we receive 27,491 observations from 2685 unique individuals. We apply poststratification weights so that the sample represents the Austrian population between 14-75 years. Question wordings, further details on the construction of the additive index, and information on nonresponse and panel attrition are given in Appendix A \& E.

Second, we use data on Austrian-wide mobility during the COVID-19 pandemic observed from mobile phones based on the location of GSM network base stations they are assigned to. Anonymized data from the GSM network was provided by a large Austrian internet service provider (ISP). Our analyses are based on aggregated data containing daily mobility estimates by age-group, gender, and region. These aggregates rely on a micro-dataset that registers events for every call as well as data communications of approximately 1.2 million devices registered with the partner ISP (Heiler et al. 2020). Local data privacy regulations have been met and the recommendations of the alliance of mobile phone providers have been followed. To estimate individual mobility, we use the radius of gyration (ROG), which is defined as the time-weighted root mean square distance between the center of gravity (i.e. the coordinate-wise time-weighted average), and each localization. This measure is 
commonly used to estimate mobility patterns of the general population (González et al. 2008; Pappalardo et al. 2015; Song et al. 2010). The distribution of the ROG is heavily skewed. To mitigate the effect of data skew, we use the daily median ROG to calculate weekly aggregated mobility averages. As the GSM data includes information about the age group and gender of its users, we also compare GSM and survey self-reports within and between these subgroups. We weight the data so that the resulting distributions resemble the Austrian target population (aged 15-74). This approach enables us to exclude the possibility that differences in the distribution of these variables between the GSM mobility data and the survey data bias our mobility estimates. For further details on the GSM-data and the calculation of ROG refer to Appendix A.

Third, we use data from the COVID-19 Community Mobility Reports published by Google (Google 2021a). This dataset provides aggregated daily movement trends by region across six different types of places: grocery stores and pharmacies, parks, transit stations, retail and recreation areas, residential areas, and workplaces. Google specifies that these reports are created with aggregated, anonymized sets of data from users who have turned on the 'Location History' setting. Besides GPS data, Google's Location History service uses further information derived from WiFi and mobile network. However, Google provides no further details on the measure itself except that it describes peoples' "visits and length of stay" (Google 2021b). Thus, this study also provides a benchmark for how this measure compares to other estimates of mobility. Google mobility data is reported as a percent change to a baseline which is the median value from the five weeks from January $3^{\text {rd }}$ to February $6^{\text {th }}, 2020$. We calculate an additive index of all six different mobility categories and estimate weekly averages. The differentiation of places also allows us to compare specific mobility trends to specific self-reported frequencies in the survey. To do that, we calculate an additional additive index summing survey answers on the frequency of 'buying medicine or receiving medical treatments' and 'buying food' and compare it to the mobility reported in Google's 'grocery \& pharmacy' category. Furthermore, we compare survey answers on 'buying non-food products' to Google's 'retail \& recreation' category and 'work' to Google's 'workplace' category.

We focus on the detection of mobility trends, representing one of the main goals in mobility research during the pandemic, and compare them between data sources. Due to the heterogeneous measurements and scales, a direct comparison of these mobility estimates is challenging. Hence, this paper investigates similarities and differences of the three data types in aggregate relative changes in mobility trends over time. We define the first wave of the survey as our baseline week (March 23 - 29, 2020) and calculate relative percentage changes from this baseline for all three data sources (we provide additional checks varying the baseline in Appendix B). Afterward, we match weekly mobility averages in the GSM and Google data to the respective survey waves (calculating a 7-day moving average results in highly similar estimates see Appendix A). Thus, we compare relative changes in the country-wide aggregates of self-reported mobility, GSM-mobility, and Google's mobility reports. To test the similarity of aggregated mobility trends between different mobility estimates, we calculate Pearson's correlations and trend lines. In addition, we provide robustness checks utilizing linear regression with time fixed-effects and a difference in difference analysis. 


\section{Results}

\section{General trends across different mobility estimates}

Figure 1 shows the trends in mobility patterns using three different sources to estimate mobility in Austria: GPS, GSM, and surveys. In general, the trends of all three mobility estimates are quite similar. After the first lockdown, mobility rose until it reached its peak in summer 2020 (our baseline week is the last week of March 2020, one week after the beginning of the first lockdown in Austria). After the first restrictions have been re-introduced in September, mobility started to decrease. This decreasing trend had been relatively consistent until mid of January when mobility slowly begun to rise again. However, some differences between the estimates remain: The GSM estimated mobility pattern clearly shows the largest variation over time, which might be a result of the different measurement strategies: while the GSM based mobility data estimates absolute physical mobility, the survey data comprise self-reported frequencies of certain types of mobility, resulting in the least amount of variation over time among the three measures. Google's mobility estimates seem to capture a middle-ground with more substantial variation than the survey estimates, but lower volatility than the absolute mobility estimates of the GSM. This suggests that - in line with Google's specifications - this estimate combines people's frequencies of movements and duration of stay rather than measuring their (more volatile) absolute mobility.

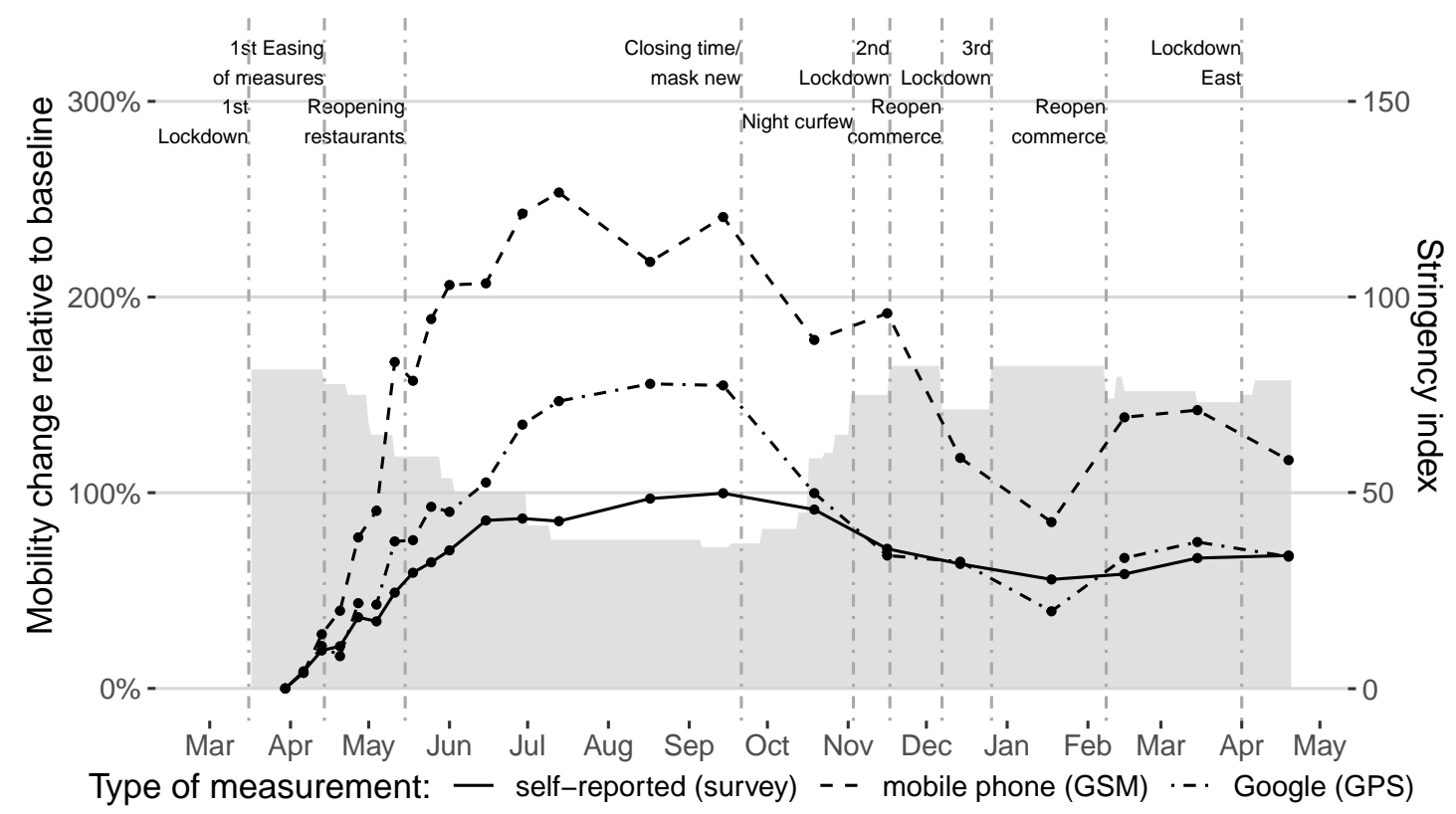

Figure 1: Mobility trends in Austria. The black lines represents average mobility change relative to the baseline (Mar 23 - 29, 2020). The gray area indicates the COVID-19 stringency index[0-100] (containment and closure policies) for Austria (Hale et al. 2021).

\section{Mobility trends within and between subgroups}

To get a more nuanced picture of the similarities between the changes in survey mobility estimates and the two mobile phone based mobility estimates over time, we focus on the 
correlations between these estimates. Pearson's correlation coefficient provides a basic measure, whether aggregate mobility estimates within groups follow a similar time trend in both compared mobility measures. The high correlation between survey self-reports and estimated behavior from GSM data confirms the notion of strongly similar time trends $(\mathrm{R}=0.927, \mathrm{p}<0.001,2$-sided $)$. Figure 2 shows correlation coefficients remain high when differentiating by gender and age. The similar slopes in Panel (2a) indicate that differences in the mobility patterns between genders across data sources are consistent. This is not the case when looking at different age groups. The larger slopes of the fitted trend lines (2b) for the youngest and the oldest respondents in the sample indicate that these groups show more variation in the survey estimates over time compared to middle-aged respondents (30 to 59 years). This is also supported by regression estimates reported in Appendix D. One explanation for these differences might be the stronger work obligations for middle-aged individuals: as work-related mobility was still permitted during the lockdown, workers could reduce their mobility less than other groups in society. This results in lower relative increases in the summer for this age group especially within the survey data (see also relative changes over time in Appendix B). Differences between the estimates might emerge because increases in commuting should have smaller effects on the reported frequency of mobility in the survey estimate compared to the GSM estimate that focuses on absolute distances.

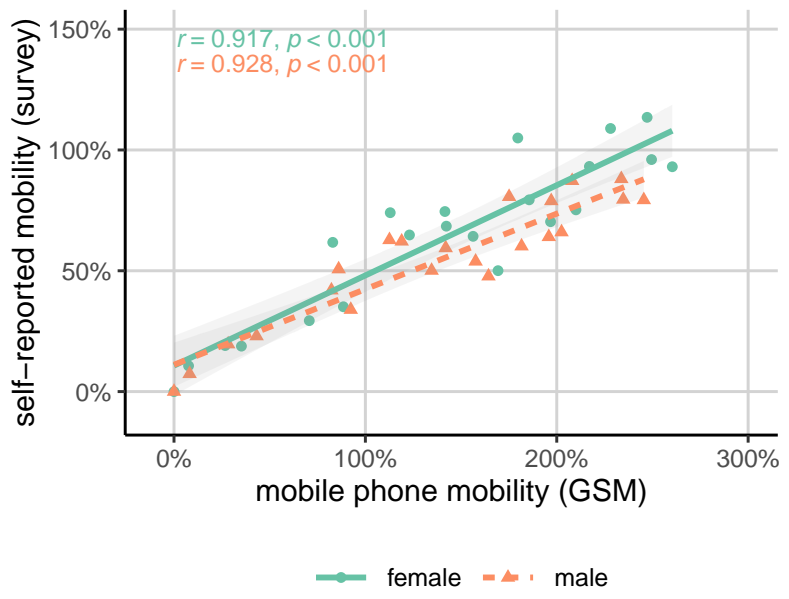

(a) By gender

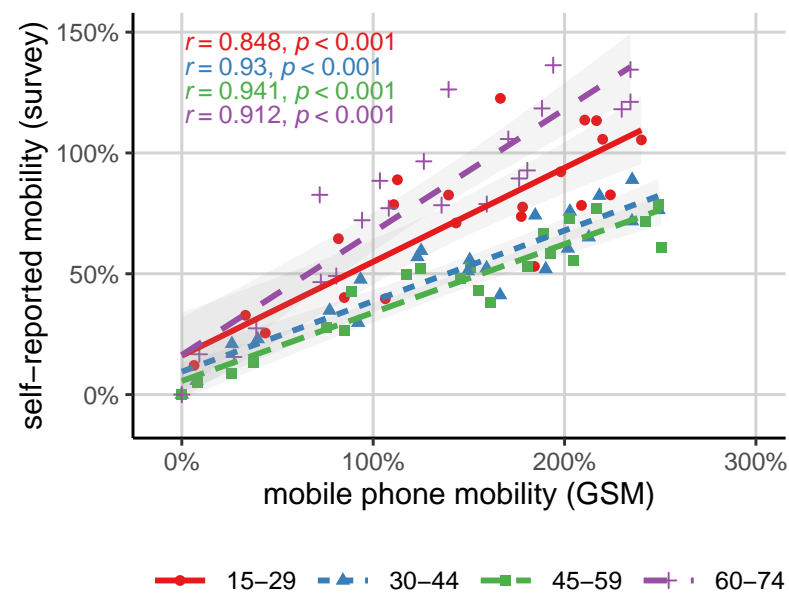

(b) By age group

Figure 2: Correlation of mobility estimates (averages by subgroup and week). P values refer to two-sided tests for statistical significance.

Figure 3 provides correlations between aggregate measures of frequencies on leaving the house for different reasons (self-reported) and the frequency of movement in different place categories (Google GPS). The correlation coefficients suggest high similarities within subgroup trends over time. However, the different slopes indicate different strengths in the relationships between the survey and Google's GPS-based mobility estimates. While the mobility estimate of the category 'shopping (food and medicine)' nearly follows a 1:1 ratio, the mobility estimate of 'shopping (other)' more than quadruples over time in the GPS estimates and 
only doubles in the survey estimate. Separate analyses for survey-related mobility to buy food or medicine suggest little differences in comparison to the combined index. Contrary, work-related mobility increases by about $150 \%$ in the survey data, while the GPS estimate only doubles over time. Again, these results indicate substantial similarity in trends within subgroups, while differences between subgroups across the different mobility measures can be substantial. An important reason for this finding is the low amount of mobility in Google's estimates related to 'shopping (other)' (Google's 'retail \& recreation' place category) during the start of the pandemic (see changes over time in Appendix B). Since take-away and pick-up were permitted during the lockdown, and Google's mobility indicator also considers how long people stay in these places, the technology might underestimate shopping-related mobility in this period. Moreover, it is likely that Google's place category captures a wider range of mobility than our corresponding survey estimate.

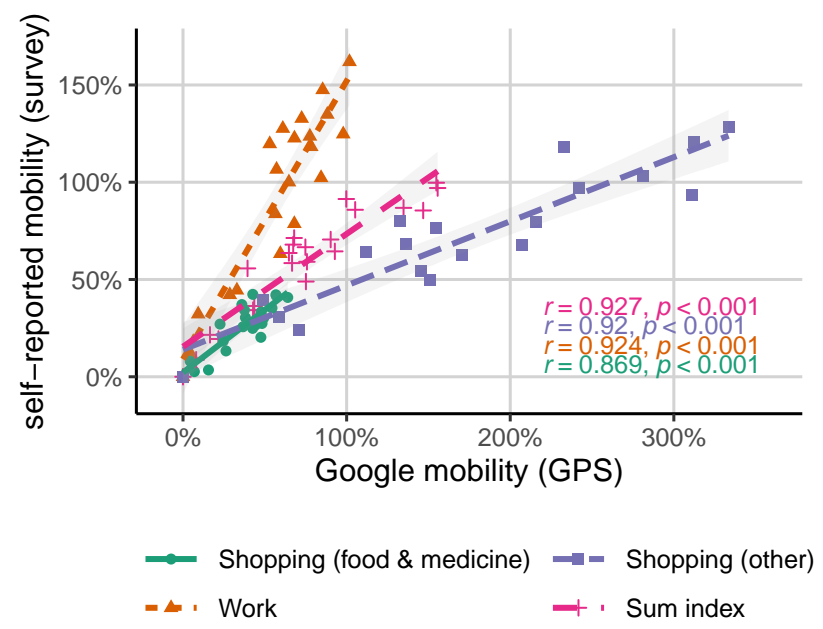

Figure 3: Correlation of mobility estimates (averages by category and week). P values refer to two-sided tests for statistical significance.

\section{Robustness Checks}

To check the robustness of these results, we also provide a regression based approach to compare the aggregated mobility estimates. These models use time-fixed effects and the estimates generally support the findings reported above (see Appendix E). Furthermore, we calculated mobility patterns using a second mobility estimate that utilizes respondents' self-reported frequency of staying at home. We compare these to GSM-data estimates indicating the share of people having a ROG of more than 500 meters. Overall, the results are qualitatively similar to those reported above (refer to Appendix C). To further substantiate our finding that mobility patterns of these different estimates are qualitatively similar and capture at least closely related latent variables, we test the impact of a partial lockdown in Austria using a differences-in-differences approach. Results (reported in Appendix D) indicate that all three measures are capturing substantial decreases in mobility due to the lockdown. 


\section{Discussion and conclusion}

Using data from Austria, this paper provides a comparison of mobility estimates from three main data sources GPS, GSM, and survey data. Our findings show that all three mobility estimates change similarly over time and portray similar trends within socio-demographic subgroups. Although all three data sources might produce different and potentially biased point estimates of mobility due to differences in representation and measurement, researchers interested in over-time changes of mobility estimates should receive comparable results. Thus, surveys can indeed help to explain mobility patterns obtained from mobile phone data, and, vice versa, mobile phone data can substantiate self-reported behavior obtained from surveys.

Our findings also support other results in the literature suggesting that survey estimates measuring mobility and compliance with mobility restrictions show rather low levels of social desirability bias (Larsen et al. 2020; Jensen 2020; Daoust et al. 2020). In fact, when policy stringency decreases, we find that our self-reported mobility measure increases to a lesser degree than the other mobility estimates (see also Appendix E). Furthermore, using smartphone data directly linked to survey data, Gollwitzer and colleagues (2020) report high correlations between self-reported social distancing and actual movement data on both the individual and the state level. Our findings suggest that this result also holds over a long period spanning several different phases of the COVID-19 pandemic, and also persists when focusing on time trends specific to gender and age groups or different mobility categories.

However, while our results indicate that mobility measures using different estimation strategies are quite comparable, our findings also highlight some differences: First, while trends are similar, the size of variation over time is not. Especially the GSM measure using data on absolute mobility produces more variation over time compared to the other mobility measures. Second, different mobility measures have different subgroup-specific variation over time. Our findings suggest that variation in work mobility over time should be more pronounced in the GSM measure, followed by the survey-based indicator, and finally by Google's Mobility Reports. Hence, a simple comparison would suggest three different results for the relative importance of work for overall mobility changes during the COVID-19 pandemic. Researchers should be aware of the specific forms of mobility that different sources of mobility estimates capture and acknowledge differences between measures that target frequency of mobility, absolute mobility, or physical distance.

These issues of construct validity, targeting the 'inferential leap' between the phenomenon of interest and the data source (Conrad, Keusch, and Schober 2021), are crucial besides the commonly mentioned issues of measurement and representation errors, which remain important even in Big Data contexts (Amaya, Biemer, and Kinyon 2020). However, despite specific sources of errors in GPS, GSM, and survey data sources, all seem capable to measure mobility patterns during the COVID-19 pandemic. This highlights especially the power of research designs that rely on estimate changes over time. Nevertheless, more efforts in combining these measures at the micro-level could enhance our understanding of how different data sources capture people's behavior. 


\section{Data Availability Statement}

The survey data used in this study can be obtained here https://doi.org/10.11587/28KQNS. Google mobility reports are available here: https://www.gstatic.com/covid19/mobi lity/Region_Mobility_Report_CSVs.zip. GSM data is not available because of the permission policy of the original data collector. The authors have asked the editors to waive the journal's replication policy for this manuscript. The statistical code is available at https://osf.io/c6vn7/?view_only=faec055c10f0466a823b4bfa23e44a84.

\section{References}

Alfano, Vincenzo, and Salvatore Ercolano. 2020. "The Efficacy of Lockdown Against COVID19: A Cross-Country Panel Analysis." Applied Health Economics and Health Policy 18 (4): 509-17. https://doi.org/10.1007/s40258-020-00596-3.

Amaya, Ashley, Paul P. Biemer, and David Kinyon. 2020. "Total Error in a Big Data World: Adapting the TSE Framework to Big Data." Journal of Survey Statistics and Methodology 8 (1): 89-119. https://doi.org/10.1093/jssam/smz056.

Bavel, Jay J. Van, Katherine Baicker, Paulo S. Boggio, Valerio Capraro, Aleksandra Cichocka, Mina Cikara, Molly J. Crockett, et al. 2020. "Using Social and Behavioural Science to Support COVID-19 Pandemic Response." Nature Human Behaviour 4 (5): 460-71. https://doi.org/10.1038/s41562-020-0884-z.

Buckee, Caroline O., Satchit Balsari, Jennifer Chan, Mercè Crosas, Francesca Dominici, Urs Gasser, Yonatan H. Grad, et al. 2020. "Aggregated Mobility Data Could Help Fight COVID-19." Science 368 (6487): 145-46. https://doi.org/10.1126/science.abb8021.

Chu, Derek K., Elie A. Akl, Stephanie Duda, Karla Solo, Sally Yaacoub, Holger J. Schünemann, Derek K. Chu, et al. 2020. "Physical Distancing, Face Masks, and Eye Protection to Prevent Person-to-Person Transmission of SARS-CoV-2 and COVID-19: A Systematic Review and Meta-Analysis." The Lancet 395 (10242): 1973-87. https://doi.org/10.1016/S01406736(20)31142-9.

Conrad, Frederick G., Florian Keusch, and Michael F. Schober. 2021. "New Data in Social and Behavioral Research." Public Opinion Quarterly (nfab027). https://doi.org/10.1093/po q/nfab027.

Daoust, Jean-François, Richard Nadeau, Ruth Dassonneville, Erick Lachapelle, Éric Bélanger, Justin Savoie, and Clifton van der Linden. 2020. "How to Survey Citizens' Compliance with COVID-19 Public Health Measures: Evidence from Three Survey Experiments." Journal of Experimental Political Science, 1-8. https://doi.org/10.1017/XPS.2020.25.

Gauvin, Laetitia, Michele Tizzoni, Simone Piaggesi, Andrew Young, Natalia Adler, Stefaan Verhulst, Leo Ferres, and Ciro Cattuto 2020. "Gender Gaps in Urban Mobility." Humanities and Social Sciences Communications 7 (1): 1-13. https://doi.org/10.1057/s41599-020-0500-x. 
Gollwitzer, Anton, Cameron Martel, Julia Marshall, Johanna Marie Höhs, and John A. Bargh. 2020. "Connecting Self-Reported Social Distancing to Real-World Behavior at the Individual and U.S. State Level." PsyArXiv. https://doi.org/10.31234/osf.io/kvnwp.

González, Marta C., César A. Hidalgo, and Albert-László Barabási. 2008. "Understanding Individual Human Mobility Patterns." Nature 453 (7196): 779-82. https://doi.org/10.1038/ nature06958.

Google. 2021a. "COVID-19 Community Mobility Report." COVID-19 Community Mobility Report. https://www.google.com/covid19/mobility?hl=en.

2021b. "Overview - Community Mobility Reports Help." https://support.google.com /covid19-mobility/answer/9824897?hl=en\&ref_topic=9822927\#zippy=.

. 2022a. "How Google Uses Location Information." https://policies.google.com/techno logies/location-data?hl=en.

. 2022b. "Mobility Data Report CSV Documentation." https://www.google.com/covid 19/mobility/data_documentation.html?hl=en.

Grantz, Kyra H., Hannah R. Meredith, Derek A. T. Cummings, C. Jessica E. Metcalf, Bryan T. Grenfell, John R. Giles, Shruti Mehta, et al. 2020. "The Use of Mobile Phone Data to Inform Analysis of COVID-19 Pandemic Epidemiology." Nature Communications 11 (1): 4961. https://doi.org/10.1038/s41467-020-18190-5.

Hale, Thomas, Noam Angrist, Rafael Goldszmidt, Beatriz Kira, Anna Petherick, Toby Phillips, Samuel Webster, Emily Cameron-Blake, Laura Hallas, Saptarshi Majumdar, and Helen Tatlow. 2021. "A Global Panel Database of Pandemic Policies (Oxford COVID-19 Government Response Tracker)." Nature Human Behaviour 5(4): 529-38. https://doi.org/10.1038/s41562021-01079-8.

Haug, Nils, Lukas Geyrhofer, Alessandro Londei, Elma Dervic, Amélie Desvars-Larrive, Vittorio Loreto, Beate Pinior, Stefan Thurner, and Peter Klimek. 2020. "Ranking the Effectiveness of Worldwide COVID-19 Government Interventions." Nature Human Behaviour 4 (12): 1303-12. https://doi.org/10.1038/s41562-020-01009-0.

Heffner, Joseph, Marc-Lluís Vives, and Oriel FeldmanHall. 2021. "Emotional Responses to Prosocial Messages Increase Willingness to Self-Isolate During the COVID-19 Pandemic." Personality and Individual Differences 170 (February): 110420. https://doi.org/10.1016/j.pa id.2020.110420.

Heiler, Georg, Tobias Reisch, Jan Hurt, Mohammad Forghani, Aida Omani, Allan Hanbury, and Farid Karimipour. 2020. "Country-Wide Mobility Changes Observed Using Mobile Phone Data During COVID-19 Pandemic." arXiv:2008.10064. http://arxiv.org/abs/2008.10064.

Jay, Jonathan, Jacob Bor, Elaine O. Nsoesie, Sarah K. Lipson, David K. Jones, Sandro 
Galea, and Julia Raifman. 2020. "Neighbourhood Income and Physical Distancing During the COVID-19 Pandemic in the United States." Nature Human Behaviour 4 (12): 1294-1302. https://doi.org/10.1038/s41562-020-00998-2.

Jensen, Ulrich Thy. 2020. "Is Self-Reported Social Distancing Susceptible to Social Desirability Bias? Using the Crosswise Model to Elicit Sensitive Behaviors." Journal of Behavioral Public Administration 3 (2). https://doi.org/10.30636/jbpa.32.182.

Jordan, Jillian, Erez Yoeli, and David Rand. 2020. "Don't Get It or Don't Spread It? Comparing Self-Interested Versus Prosocial Motivations for COVID-19 Prevention Behaviors". https://doi.org/10.31234/osf.io/yuq7x.

Keusch, Florian, Bella Struminskaya, Christopher Antoun, Mick P Couper, and Frauke Kreuter. 2019. "Willingness to Participate in Passive Mobile Data Collection." Public Opinion Quarterly 83 (S1): 210-35. https://doi.org/10.1093/poq/nfz007.

Kitamura, Shuhei, and Katsunori Yamada. 2020. "Social Comparisons and Cooperation During COVID-19." PsyArXiv. https://doi.org/10.31234/osf.io/rsbmz.

Kittel, Bernhard, Sylvia Kritzinger, Hajo Boomgaarden, Barbara Prainsack, Jakob-Moritz Eberl, Fabian Kalleitner, Noëlle S. Lebernegg, et al. 2020. "The Austrian Corona Panel Project: Monitoring Individual and Societal Dynamics Amidst the COVID-19 Crisis." European Political Science 20: 318-44. https://doi.org/10.1057/s41304-020-00294-7.

Kittel, Bernhard, Sylvia Kritzinger, Hajo Boomgaarden, Barbara Prainsack, Jakob-Moritz Eberl, Fabian Kalleitner, Noëlle S. Lebernegg, Julia Partheymüller, Carolina Plescia, David W. Schiestl, Lukas Schlogl. 2020. "Austrian Corona Panel Project (SUF edition)" AUSSDA, V5, https://doi.org/10.11587/28KQNS UNF:6:gjUyW1lm3U433cVw4RK47g==.

Kittel, Bernhard, Fabian Kalleitner, and David W. Schiestl. 2021. "Avoiding a Public Health Dilemma: Social Norms and Trust Facilitate Preventive Behaviour If Individuals Perceive Low COVID-19 Health Risks". https://doi.org/10.31235/osf.io/q9b23.

Larsen, Martin, Jacob Nyrup, and Michael Bang Petersen. 2020. "Do Survey Estimates of the Public's Compliance with COVID-19 Regulations Suffer from Social Desirability Bias?" Journal of Behavioral Public Administration 3 (2). https://doi.org/10.30636/jbpa.32.164.

Oliver, Nuria, Bruno Lepri, Harald Sterly, Renaud Lambiotte, Sébastien Deletaille, Marco De Nadai, Emmanuel Letouzé, et al. 2020. "Mobile Phone Data for Informing Public Health Actions Across the COVID-19 Pandemic Life Cycle." Science Advances 6 (23): eabc0764. https://doi.org/10.1126/sciadv.abc0764.

Pappalardo, Luca, Filippo Simini, Salvatore Rinzivillo, Dino Pedreschi, Fosca Giannotti, and Albert-László Barabási. 2015. "Returners and Explorers Dichotomy in Human Mobility." Nature Communications 6 (1): 8166. https://doi.org/10.1038/ncomms9166. 
Pepe, Emanuele, Paolo Bajardi, Laetitia Gauvin, Filippo Privitera, Brennan Lake, Ciro Cattuto, and Michele Tizzoni. 2020. "COVID-19 Outbreak Response, a Dataset to Assess Mobility Changes in Italy Following National Lockdown." Scientific Data 7 (1): 230. https: //doi.org/10.1038/s41597-020-00575-2.

Pfattheicher, Stefan, Laila Nockur, Robert Böhm, Claudia Sassenrath, and Michael Bang Petersen. 2020. "The Emotional Path to Action: Empathy Promotes Physical Distancing and Wearing of Face Masks During the COVID-19 Pandemic." Psychological Science 31 (11): 1363-73. https://doi.org/10.1177/0956797620964422.

Rojas, Mario B., Eazaz Sadeghvaziri, and Xia Jin. 2016. "Comprehensive Review of Travel Behavior and Mobility Pattern Studies That Used Mobile Phone Data." Transportation Research Record 2563 (1): 71-79. https://doi.org/10.3141/2563-11.

Schlosser, Frank, Benjamin F. Maier, Olivia Jack, David Hinrichs, Adrian Zachariae, and Dirk Brockmann. 2020. "COVID-19 Lockdown Induces Disease-Mitigating Structural Changes in Mobility Networks." Proceedings of the National Academy of Sciences 117 (52): 32883-90. https://doi.org/10.1073/pnas.2012326117.

Schwarz, Norbert. 2007. "Cognitive Aspects of Survey Methodology." Applied Cognitive Psychology 21 (2): 277-87. https://doi.org/10.1002/acp.1340.

Song, Chaoming, Zehui Qu, Nicholas Blumm, and Albert-László Barabási. 2010. "Limits of Predictability in Human Mobility." Science 327 (5968): 1018-21. https://doi.org/10.1126/sc ience.1177170.

Struminskaya, Bella, Vera Toepoel, Peter Lugtig, Marieke Haan, Annemieke Luiten, and Barry Schouten. 2021. "Understanding Willingness to Share Smartphone-Sensor Data." Public Opinion Quarterly, no. nfaa044 (February). https://doi.org/10.1093/poq/nfaa044.

Vinceti, Marco, Tommaso Filippini, Kenneth J. Rothman, Fabrizio Ferrari, Alessia Goffi, Giuseppe Maffeis, and Nicola Orsini. 2020. "Lockdown Timing and Efficacy in Controlling COVID-19 Using Mobile Phone Tracking." EClinicalMedicine 25: 100457. https://doi.org/10 .1016/j.eclinm.2020.100457.

Vollmer, M, S Mishra, H Unwin, A Gandy, T Melan, V Bradley, H Zhu, et al. 2020. "Report 20: A Sub-National Analysis of the Rate of Transmission of Covid-19 in Italy." Imperial College London. https://doi.org/10.25561/78677.

Zhang, Mengxi, Siqin Wang, Tao Hu, Xiaokang Fu, Xiaoyue Wang, Yaxin Hu, Briana Halloran, Yunhe Cui, Haokun Liu, Zhimin Liu, Shuming Bao. 2020. "Human mobility and COVID-19 transmission: a systematic review and future directions." MedRxiv. https: //doi.org/10.1101/2021.02.02.21250889. 


\section{Appendix}

\section{A) Further details on the data sources and the computation of mobility measures}

\section{Survey data}

The panel survey provided by the Austrian Corona Panel Project (ACPP) uses non-probability sampling relying on a commercial online access panel provided by Marketagent.com (certified under ISO 20252). The target population includes Austrian residents with at least 14 years of age and access to the internet. The surveys were administered using web panel invitations send out via E-Mail. One E-Mail reminder is sent 4 days after the initial invitation in each wave. The panel utilizes quota sampling based on the following variables and categories: gender (male, female); age (14-19, 20-29, 30-39, 40-49, 50-59, 60-69, 70-99); region (the nine Austrian provinces 'Bundesländer'); municipality size (<5.000 inhabitants, 5.000 - 50.000, 50.000 - 1 million; $>1$ million); and highest level of education (ISCED 0-3; ISCED >3= upper secondary education or higher). The original questionnaire was fielded in German. The survey has no information about respondent's location while filling in the survey. Thus, we cannot exclude respondents on holiday (answers to questions about the plans for summer holidays in 2020 , however, suggest that less than $1.7 \%$ of respondents should be on holiday during each of the survey's field periods).

The announced survey duration is 18 minutes and respondents receive 180 'credit points' as compensation after completing the survey which can be exchanged to $1,80 €$ in cash or coupons. The initial response rate (AAPOR RR1) in the first survey wave was 35.2\% (1541 interviews after 4381 invitations). On average 1,547 respondents per wave completed the survey and panelists participated on average in 11.9 of the 22 survey waves. Response rates to replace drop outs varied between 2.4\% (July 2020) and 8.1\% (May 2020). All who participate in the survey were invited to participate again in subsequent waves at least once. Response rates of subsequent waves started at $86.2 \%$ and dropped afterwards, stabilizing after wave 12 at around $60 \%$. On average, 58 participants per wave were invited to replace drop outs (0 interviews in wave 20 and $21-231$ in wave 2 ). Information on the field periods can be accessed on the original project website (ACPP 2021).

We use post-stratification weights that are provided alongside the official survey release. Weights were calculated using data about the distributions of gender, age-groups, level of education, region and gender $\mathrm{X}$ age-group provided by the Austrian federal agency for statistics (Statistik Austria 2019). To verify that the same respondents answered each survey wave, basic socio-demographics (age, gender, region) were asked in every survey wave and cross validated with the previous answers.

As a non-probability survey, the data has several limitations. First, statistical tests rely on the assumption that participants are randomly chosen from a defined set of participants (see

e.g. Vehovar 2016). Thus, the quality of the survey-sample is dependent on the quality of panelists registered with the specific commercial online access panel in representing the target 
population. Marketagent.com has quite a large sample pool in Austria (129,500 registered panelists) and uses online and offline recruiting, including advertising on television, print media, radio, and billboards. This should reduce potential coverage errors. Second, utilizing web mode only excludes part of the population by design. While still relevant to some degree, this issue has become less crucial in the last years, and coverage error should be smaller in our target group (14-74) compared to the general population (in 2021, 95\% of Austrian households with at least one household member aged between 16 and 74 years had internet access (Statistik Austria 2021). In sum, these issues suggest that the digitally skilled population might be overrepresented in our survey. This could make our survey based mobility estimates more similar to the estimates provided by Google's mobility reports and the GSM data compared to traditional probability based surveys. Our main comparison focuses on change over time, thus consistency within the sample distribution should be most crucial. This highlights the issue of panel attrition.

To test whether panel attrition could have affected the survey mobility estimates, we check which respondents left the survey and whether our resampled subjects have similar mobility characteristics. To test this, we assume that those who left the panel and those resampled should have similar differences in mobility behavior compared to the panelists remaining in the survey. This is described in equation (1) calculating the mobility measure mobility for each individual $i$. Where part $_{i, t-1}$ is a dummy variable indicating if a participant participated in the previous wave $t-1$ and part $_{i, t}$ is a dummy variable indicating if a participant was present in the current survey wave $t$. We calculate separate models for each possible pair of survey waves and compare the differences in the two dummy variables using a linear hypothesis test (2019). The basic idea is that those who dropped out and those who are resampled should have roughly similar differences in mobility compared to those who are present in both waves. Hence the two dummy variables should be roughly equal in size (formally $\beta_{1}=\beta_{2}$ ).

$$
\text { mobility }_{i}=\beta_{1} * \text { part }_{i, t-1}+\beta_{2} * \text { part }_{i, t}+\beta_{3} * \text { wave }_{i, t}+\alpha+\epsilon_{i}
$$

We find that the average difference between these two coefficients is quite small (0.0118) and get 16 statistically significant differences out of $215 \mathrm{p}<0.05$ and 29 for $\mathrm{p}<0.1$. These shares are roughly equal to what would we expect because of false positives. This suggests that those joining or rejoining the sample have similar differences to those those who stay in the sample. Aggregate comparisons between waves should therefore provide quite consistent results if the assumption holds that the initial selection of participants into the survey is independent of patterns of change in the population. We note that these differences are not only small because similar panellists were re-sampled but also because the average mobility of those who dropped out did not differ much from those who remained in the sample $\left(\overline{\beta_{1}}=0.023\right)$.

To check for potential biases because of item-nonresponse we first asses the shares of missing values for our aggregate mobility estimate and each mobility component. Afterwards we calculate binary logistic regression models with wave fixed-effects predicting the log of the odds of respondents providing missing values for our aggregate mobility estimate and each mobility component focusing on our main socio-demographic variables of interest in the 
manuscript (gender and age). On average, our main aggregate mobility measure misses for $15.90 \%$ of respondents each wave. The missing values are distributed quite equally across the components of the additive index with exception of the answer category 'other reasons' ( work $=3.20 \%$, sport $=2.22 \%$, friends $=2.35 \%$, medicine $=2.51 \%$, food $=1.78 \%$, pets $=4.38 \%$, pets $=2.99 \%$, boredom $=4.43 \%$, other $=11.2 \%$ ). Dropping the variable 'other reason' from our mobility index would reduce average misses for our aggregate measure to $9.58 \%$. However, using this measure we receive nearly equivalent relationships over time to the GSM (Pearson's $\mathrm{R}=0.931$ ) and Google GPS-based data (Pearson's $\mathrm{R}=0.928$ ) compared to the results reported in the main manuscript using the full range of variables. This is because, on an individual level, the 'other' category does not add much variance compared to the index excluding this variable (Pearson's $\mathrm{R}=0.983$ ) and it seems that those who did avoid responding to this category did not change differently from those who answered.

Focusing on regression results provided in table 1, we find less missing values for middle aged respondents (45-59) compared to the youngest age cohort (14-29). Looking more closely at the patterns, we find some indications that people might use 'don't know' or 'no answer' options to indicate no mobility in this category, as e.g. males and those in the working age population have less missing values in the work category than others. Hence, we would suggest that structurally similar questions in the future could profit from more clear indication of the answer option corresponding to zero mobility. We also find more item non-response between waves 13 and 19 and in wave 22. This could indicate some fatigue effects or that increased mobility during summer months lead some respondents to choose to avoid an answer here. All in all, however, the effects are rather small in size. For instance, the odds for middle aged respondents $(45-59)$ to have missing values are $12 \%$ smaller $(\exp (-0.127)=0.881)$ than for the reference group. Hence, ceteris paribus, we would expect $16.9 \%$ missing values for middle aged respondents compared to $19.2 \%$ for respondents in the youngest age group (14-29).

Survey field periods were not strictly aligned with calendar weeks and the duration varied depending on how fast the sample reached the target of 1500 respondents per wave. We therefore had to decide upon reference points determining the week used to calculate averages of the mobile phone (GSM) and Google mobility (GPS) data. As the median response of the survey only varies from Friday to Sunday, with most field phases starting on a Friday, we decided to use the date of the median response as our indicator for the baseline week. Hence, if the median response in the survey was in week 19, we utilize the average daily movement measures of calendar week 19 in the other measures. This is a compromise due to the varying reference period in the survey ('last 7 days'). As this reference point depends on the date the respondents answered the survey, one would have to match the right mobility data to the right respondents, which is not possible in our case. A running average approach, matching every survey respondent (irrespective of other characteristics) with a corresponding 7-day average according to the day they finished the survey and calculating mean values afterwards, produces nearly no differences with regard to the main results of interest (Pearson's correlation coefficients: ACPP-GSM $=0.935 ; \mathrm{ACPP}-\mathrm{GOG}=0.935 ; \mathrm{GSM}-\mathrm{GOG}=0.955)$. We suggest that other studies might consider calendar-based reference periods like "mobility in the week of data $\mathrm{x}$ to date $\mathrm{y}$ " in their survey questions if field periods increase in time. 
Table 1: Regression estimates of a binary logistic regression on respondents likelihood of item-nonresponse

\begin{tabular}{|c|c|c|c|c|c|c|c|c|c|c|}
\hline & \multicolumn{10}{|c|}{ Item nonresponse: } \\
\hline & $\begin{array}{l}\text { Index } \\
(1)\end{array}$ & $\begin{array}{l}\text { work } \\
(2)\end{array}$ & $\begin{array}{l}\text { sport } \\
(3)\end{array}$ & $\begin{array}{l}\text { friends } \\
(4)\end{array}$ & $\begin{array}{c}\text { medicine } \\
(5)\end{array}$ & $\begin{array}{l}\text { food } \\
(6)\end{array}$ & $\begin{array}{l}\text { pets } \\
(7)\end{array}$ & $\begin{array}{c}\text { bordom } \\
(8)\end{array}$ & $\begin{array}{c}\text { shopping } \\
(9)\end{array}$ & $\begin{array}{l}\text { other } \\
(10)\end{array}$ \\
\hline $\begin{array}{l}\text { Gender: male } \\
\text { (ref. female) }\end{array}$ & $\begin{array}{c}-0.038 \\
(0.030) \\
p=0.209\end{array}$ & $\begin{array}{c}-0.129 \\
(0.063) \\
p=0.042\end{array}$ & $\begin{array}{c}0.109 \\
(0.076) \\
p=0.150\end{array}$ & $\begin{array}{c}0.091 \\
(0.074) \\
p=0.216\end{array}$ & $\begin{array}{c}0.153 \\
(0.071) \\
p=0.033\end{array}$ & $\begin{array}{c}0.153 \\
(0.085) \\
p=0.072\end{array}$ & $\begin{array}{c}0.071 \\
(0.054) \\
p=0.193\end{array}$ & $\begin{array}{c}0.150 \\
(0.066) \\
p=0.023\end{array}$ & $\begin{array}{r}0.0005 \\
(0.058) \\
p=0.994\end{array}$ & $\begin{array}{c}-0.095 \\
(0.035) \\
p=0.008\end{array}$ \\
\hline $\begin{array}{l}\text { Age: } 30-44 \\
\text { (ref:14-29) }\end{array}$ & $\begin{array}{c}-0.025 \\
(0.044) \\
p=0.560\end{array}$ & $\begin{array}{c}-0.214 \\
(0.088) \\
p=0.015\end{array}$ & $\begin{array}{c}-0.029 \\
(0.091) \\
p=0.748\end{array}$ & $\begin{array}{c}-0.093 \\
(0.088) \\
p=0.288\end{array}$ & $\begin{array}{c}0.020 \\
(0.085) \\
p=0.817\end{array}$ & $\begin{array}{c}-0.140 \\
(0.097) \\
p=0.150\end{array}$ & $\begin{array}{c}-0.009 \\
(0.073) \\
p=0.907\end{array}$ & $\begin{array}{c}0.143 \\
(0.083) \\
p=0.084\end{array}$ & $\begin{array}{c}0.178 \\
(0.073) \\
p=0.015\end{array}$ & $\begin{array}{c}0.259 \\
(0.052) \\
p=0.00000\end{array}$ \\
\hline $45-59$ & $\begin{array}{c}-0.127 \\
(0.044) \\
p=0.004\end{array}$ & $\begin{array}{c}-0.443 \\
(0.091) \\
p=0.00001\end{array}$ & $\begin{array}{c}-0.625 \\
(0.103) \\
p=0.000\end{array}$ & $\begin{array}{c}-0.624 \\
(0.098) \\
p=0.000\end{array}$ & $\begin{array}{c}-0.678 \\
(0.098) \\
p=0.000\end{array}$ & $\begin{array}{c}-0.898 \\
(0.117) \\
p=0.000\end{array}$ & $\begin{array}{c}-0.186 \\
(0.075) \\
p=0.013\end{array}$ & $\begin{array}{r}-0.352 \\
(0.090) \\
p=0.0001\end{array}$ & $\begin{array}{c}-0.504 \\
(0.083) \\
p=0.000\end{array}$ & $\begin{array}{c}0.181 \\
(0.052) \\
p=0.001\end{array}$ \\
\hline $60-74$ & $\begin{array}{c}0.031 \\
(0.045) \\
p=0.493\end{array}$ & $\begin{array}{c}-0.071 \\
(0.088) \\
p=0.421\end{array}$ & $\begin{array}{c}-1.701 \\
(0.164) \\
p=0.000\end{array}$ & $\begin{array}{c}-1.975 \\
(0.175) \\
p=0.000\end{array}$ & $\begin{array}{c}-1.854 \\
(0.164) \\
p=0.000\end{array}$ & $\begin{array}{c}-2.478 \\
(0.240) \\
p=0.000\end{array}$ & $\begin{array}{c}-0.505 \\
(0.086) \\
p=0.000\end{array}$ & $\begin{array}{c}-1.121 \\
(0.123) \\
p=0.000\end{array}$ & $\begin{array}{c}-0.969 \\
(0.103) \\
p=0.000\end{array}$ & $\begin{array}{c}0.293 \\
(0.054) \\
p=0.00000\end{array}$ \\
\hline $\begin{array}{l}\text { Wave: } 2 \\
\text { (ref. wave } 1 \text { ) }\end{array}$ & $\begin{array}{c}-0.158 \\
(0.108) \\
p=0.143\end{array}$ & $\begin{array}{c}0.470 \\
(0.312) \\
p=0.133\end{array}$ & $\begin{array}{c}0.366 \\
(0.351) \\
p=0.297\end{array}$ & $\begin{array}{c}0.055 \\
(0.315) \\
p=0.863\end{array}$ & $\begin{array}{c}0.161 \\
(0.324) \\
p=0.620\end{array}$ & $\begin{array}{c}0.602 \\
(0.397) \\
p=0.130\end{array}$ & $\begin{array}{c}-0.343 \\
(0.216) \\
p=0.112\end{array}$ & $\begin{array}{c}0.077 \\
(0.275) \\
p=0.780\end{array}$ & & $\begin{array}{c}-0.077 \\
(0.121) \\
p=0.525\end{array}$ \\
\hline 5 & $\begin{array}{r}-0.078 \\
(0.107)\end{array}$ & $\begin{array}{r}0.846 \\
(0.294)\end{array}$ & $\begin{array}{c}0.646 \\
(0.334)\end{array}$ & $\begin{array}{c}0.464 \\
(0.290)\end{array}$ & $\begin{array}{c}0.537 \\
(0.301)\end{array}$ & $\begin{array}{c}0.903 \\
(0.379)\end{array}$ & $\begin{array}{c}0.134 \\
(0.193) \\
-\quad 0.489\end{array}$ & $\begin{array}{r}0.262 \\
(0.265)\end{array}$ & $\begin{array}{r}0.093 \\
(0.215) \\
=0667\end{array}$ & $\begin{array}{r}-0.180 \\
(0.125) \\
-\quad 0149\end{array}$ \\
\hline 8 & $\begin{array}{r}p=0.463 \\
-0.047 \\
\quad(0.106) \\
p=0.659\end{array}$ & $\begin{aligned} p= & 0.005 \\
& 0.826 \\
& (0.295) \\
p= & 0.006\end{aligned}$ & $\begin{aligned} p= & 0.054 \\
& 0.897 \\
& (0.322) \\
p= & 0.006\end{aligned}$ & $\begin{aligned} p= & 0.110 \\
& 0.735 \\
& (0.277) \\
p= & 0.009\end{aligned}$ & $\begin{aligned} p= & 0.075 \\
& 0.730 \\
& (0.292) \\
p= & 0.013\end{aligned}$ & $\begin{aligned} p= & 0.018 \\
& 1.205 \\
& (0.365) \\
p= & 0.001\end{aligned}$ & $\begin{aligned} p= & 0.489 \\
& 0.106 \\
& (0.195) \\
p= & 0.587\end{aligned}$ & $\begin{aligned} p= & 0.324 \\
& 0.516 \\
& (0.253) \\
p= & 0.042\end{aligned}$ & $\begin{aligned} p= & 0.667 \\
& 0.436 \\
& (0.201) \\
p= & 0.031\end{aligned}$ & $\begin{array}{r}p=0.149 \\
-0.015 \\
(0.121) \\
p=0.902\end{array}$ \\
\hline 11 & $\begin{array}{c}0.120 \\
(0.103)\end{array}$ & $\begin{array}{c}1.045 \\
(0.286)\end{array}$ & $\begin{array}{c}0.956 \\
(0.319)\end{array}$ & $\begin{array}{c}0.565 \\
(0.285)\end{array}$ & $\begin{array}{c}0.757 \\
(0.290)\end{array}$ & $\begin{array}{c}1.237 \\
(0.364)\end{array}$ & $\begin{array}{c}0.237 \\
(0.189)\end{array}$ & $\begin{array}{c}0.512 \\
(0.253)\end{array}$ & $\begin{array}{c}0.585 \\
(0.196)\end{array}$ & $\begin{array}{c}0.170 \\
(0.116)\end{array}$ \\
\hline 14 & $\begin{aligned} p= & 0.244 \\
& 0.337 \\
& (0.099) \\
p= & 0.001\end{aligned}$ & $\begin{aligned} p= & 0.0003 \\
& 0.946 \\
& (0.289) \\
p= & 0.002\end{aligned}$ & $\begin{aligned} p= & 0.003 \\
& 0.922 \\
& (0.319) \\
p= & 0.004\end{aligned}$ & $\begin{aligned} p= & 0.048 \\
& 0.367 \\
& (0.294) \\
p= & 0.212\end{aligned}$ & $\begin{aligned} p= & 0.010 \\
& 0.778 \\
& (0.288) \\
p= & 0.007\end{aligned}$ & $\begin{aligned} p= & 0.001 \\
& 0.917 \\
& (0.377) \\
p= & 0.015\end{aligned}$ & $\begin{aligned} p= & 0.211 \\
& 0.253 \\
& (0.187) \\
p= & 0.177\end{aligned}$ & $\begin{aligned} p= & 0.043 \\
& 0.681 \\
& (0.244) \\
p= & 0.006\end{aligned}$ & $\begin{aligned} p= & 0.003 \\
& 0.384 \\
& (0.201) \\
p= & 0.057\end{aligned}$ & $\begin{aligned} p= & 0.145 \\
& 0.307 \\
& (0.113) \\
p= & 0.007\end{aligned}$ \\
\hline 17 & $\begin{array}{c}0.220 \\
(0.100) \\
p=0.029\end{array}$ & $\begin{array}{c}1.176 \\
(0.280) \\
p=0.00003\end{array}$ & $\begin{array}{c}0.721 \\
(0.328) \\
p=0.029\end{array}$ & $\begin{array}{c}0.394 \\
(0.292) \\
p=0.177\end{array}$ & $\begin{array}{c}0.630 \\
(0.294) \\
p=0.033\end{array}$ & $\begin{array}{c}1.031 \\
(0.371) \\
p=0.006\end{array}$ & $\begin{array}{c}0.231 \\
(0.187) \\
p=0.217\end{array}$ & $\begin{array}{c}0.514 \\
(0.251) \\
p=0.041\end{array}$ & $\begin{array}{c}0.538 \\
(0.195) \\
p=0.006\end{array}$ & $\begin{array}{c}0.185 \\
(0.115) \\
p=0.107\end{array}$ \\
\hline 20 & $\begin{array}{r}-0.010 \\
(0.104)\end{array}$ & $\begin{array}{c}0.799 \\
(0.294)\end{array}$ & $\begin{array}{c}1.007 \\
(0.315)\end{array}$ & $\begin{array}{c}0.698 \\
(0.277)\end{array}$ & $\begin{array}{c}0.907 \\
(0.283)\end{array}$ & $\begin{array}{c}0.880 \\
(0.379)\end{array}$ & $\begin{array}{c}0.176 \\
(0.190)\end{array}$ & $\begin{array}{c}0.595 \\
(0.248)\end{array}$ & $\begin{array}{c}0.298 \\
(0.205)\end{array}$ & $\begin{array}{c}-0.145 \\
(0.122)\end{array}$ \\
\hline 22 & $\begin{aligned} p= & 0.927 \\
& 0.297 \\
& (0.100) \\
p= & 0.003\end{aligned}$ & $\begin{aligned} p= & 0.007 \\
& 1.493 \\
& (0.272) \\
p= & 0.00000\end{aligned}$ & $\begin{aligned} p= & 0.002 \\
& 1.075 \\
& (0.313) \\
p= & 0.001\end{aligned}$ & $\begin{aligned} p= & 0.012 \\
& 0.811 \\
& (0.273) \\
p= & 0.003\end{aligned}$ & $\begin{aligned} p= & 0.002 \\
& 0.943 \\
& (0.282) \\
p= & 0.001\end{aligned}$ & $\begin{aligned} p= & 0.021 \\
& 1.013 \\
& (0.373) \\
p= & 0.007\end{aligned}$ & $\begin{aligned} p= & 0.353 \\
& 0.392 \\
& (0.183) \\
p= & 0.032\end{aligned}$ & $\begin{aligned} p= & 0.017 \\
& 0.754 \\
& (0.242) \\
p= & 0.002\end{aligned}$ & $\begin{aligned} p= & 0.146 \\
& 0.676 \\
& (0.192) \\
p= & 0.0005\end{aligned}$ & $\begin{aligned} p= & 0.237 \\
& 0.214 \\
& (0.115) \\
p= & 0.063\end{aligned}$ \\
\hline Constant & $\begin{array}{c}\quad-1.724 \\
(0.080) \\
p=0.000\end{array}$ & $\begin{array}{c}-4.209 \\
(0.251) \\
p=0.000\end{array}$ & $\begin{array}{c}-4.309 \\
(0.277) \\
p=0.000\end{array}$ & $\begin{array}{c}\quad-3.899 \\
(0.234) \\
p=0.000\end{array}$ & $\begin{array}{c}\quad-4.076 \\
(0.246) \\
p=0.000\end{array}$ & $\begin{array}{c}-4.535 \\
(0.325) \\
p=0.000\end{array}$ & $\begin{array}{c}\quad-3.175 \\
(0.150) \\
p=0.000\end{array}$ & $\begin{array}{c}-3.878 \\
(0.208) \\
p=0.000\end{array}$ & $\begin{array}{c}-3.258 \\
(0.165) \\
p=0.000\end{array}$ & $\begin{array}{c}\quad-2.277 \\
(0.093) \\
p=0.000\end{array}$ \\
\hline Observations & 32,552 & 32,552 & 32,552 & 32,552 & 32,552 & 32,552 & 32,552 & 32,552 & 28,166 & 32,552 \\
\hline
\end{tabular}

Note: Item nonresponse: $0=$ responded, 1 = avoided an answer ("Don't know" or "No answer"). Because of the space considerations we report only every third wave fixed effect. The model contains all wave dummies. Full results are available on request. Standard errors within parentheses. 
While the underlying mobility estimates from the GSM and Google data have no upper limit, the estimate obtained from the survey is limited by the 'daily' answer option. This limit, however, should not lead to substantial measurement errors due to data censoring since most people would not go to work, go shopping or do sports more often than daily. This notion is supported by the low shares of respondents choosing the answer category 'daily' (see Figure 1 below). Besides, despite this limit in the survey, the self-reported data features more work-related mobility than Google's Mobility Reports. This suggests that the issue of reduced variance in relative mobility due to an upper limit in the survey scale should be most suitable.
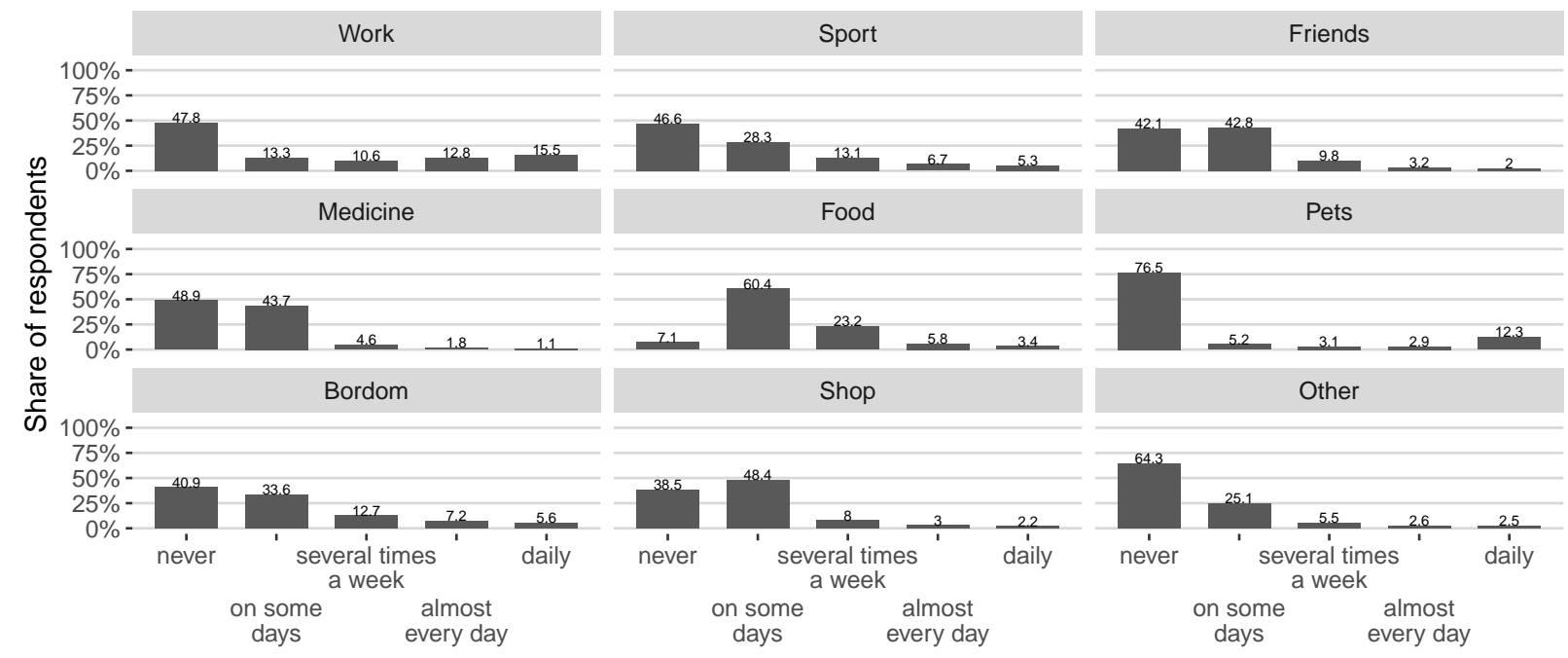

Figure 1: Answer shares of mobility items across all survey waves (by mobility category, $\mathrm{N} \approx 30.947$ per item)

The survey question on whether respondents went out to 'buy non-food products' was only asked from the fourth wave onward. This was because, until then, the governmental restrictions in Austria basically prohibited nonessential shopping. Hence, our additive index of mobility consists of 8 items until wave three, and 9 items afterwards. To account for this difference, we also exclude the place category 'retail \& recreation' for the Google estimate in the first three waves. Cronbach's alpha for the survey index on mobility is 0.74 across all 
waves, indicating that the index represents a solid construct. Dropping the items on 'work' and 'walking pets' increases alpha by only 0.02 , leading to the conclusion that the index from all items is reliable.

\section{GSM data}

The aggregated GSM data we use is based on micro data from an internet service provider (ISP) located in the medium to high price segment for telephone and internet services in Austrian business to consumer and business to business markets. The micro-dataset features a combination of classical CDR (Call Data Records) and XDR (X Data Records). Thus, the dataset not only registers an event when a call is performed but also includes additional events when data packages are transferred. On average the micro-dataset contains an event every 4 minutes for every user (this includes night times). For $80 \%$ of the events, the subsequent event is received within 1.7 minutes.

The 1.2 million devices are a sample of all customers of this ISP. The original dataset contains approximately one billion events per day from 4.5 million devices. Due to legal reasons, only those registered with the partner ISP as mobile handsets could be used. Thus, sensor devices from the Internet of Things as well as roamers and customers of virtual mobile operators are excluded. From these groups also no socio-demographic information would be available. In addition to that, customers with an opt-out for such analyses were excluded although these are quite rare $(<1 \%)$. More information on the data source can be found here (TU Wien et al. 2021) and here (Reisch et al. 2020).

According to governmental statistics in Austria. $87.8 \%$ of individuals between 16 and 74 access the internet via a smartphone in 2021. The share of users decreases with higher age: from $98.9 \%$ (16-24) to $55.5 \%$ (65-74). However, those middle aged have still quite high rates of smartphone usage (45-54 93.4\%; 55-64 79.5\%). Thus, we only expect larger coverage issues in the group of those aged 60 and older. Smartphone usage is a bit higher for males (88.9\%) 
than for females $(86.8 \%)$ and this difference increases with age $(64.5 \%, 47,6 \%)$ (Statistik Austria 2021).

The localization method is based on the topology of the network, which corresponds to the location of specific broadcasting base stations. The ISP provides the localization information for each event based on the centroid of the network coverage simulation. Thus, localization is not based on the place of the base station itself but rather on the centroid of the area where the specific base station provides the best network coverage and thus mobile devices are most likely connected to it. The literature on device-based data mainly use six types of mobility metrics (e.g., Pepe et al. 2020, Wu et al. 2021, Gauvin et al. 2020, Pappalardo et al. 2015): number of transfers between areas or provinces, number of unique destinations, stay times at locations, travel times between locations, graphical mapping of movements (e.g., heat maps), and the radius of gyration (ROG). In our case, individual mobility is calculated using the radius of gyration, which is defined as the time-weighted root mean square distance between the center of gravity (i.e. the coordinate-wise time-weighted average) and each localization. Refer to the study by Heiler et al. (2020) for more information and see e.g. the work by Gauvin et al. (2020) for a similar approach to estimating human mobility using mobile phone data.

\section{Google's GPS-based mobility reports}

The dataset is based on anonymised aggregated data from their 'Location History' feature also used to show 'Popular Times' for places in Google Maps, storing combined location data from the connected devices' antennas and sensors (next to GPS, this technology uses data from WiFi, the mobile network connection and other sensors). This feature is turned off by default and has to be activated by the users in order to be operational. In addition, the stored locations can be deleted in the user account's settings. Hence, the included userbase may not match the socio-demographic characteristics of the overall population (Google 2021a, Google 2022a, Google 2022b). An enquiry to Google on further information 
to their data stayed unanswered. In particular, we asked for details on the calculation of their measure, categorization of mobility, discrimination between types of visit to an area (e.g., work vs. shopping), discrimination between residents and foreigners, as well as the share and socio-demographic distribution of users who opted in to their 'Location History' feature. We hence cannot provide any more details on potential coverage errors in the Google data other than the specifics of smartphone use, as already explained for the GSM data.

Google's Community Mobility Reports feature six place categories: grocery and pharmacy, parks, public transport transit stations, retail and recreation, residential, and workplaces. The 'grocery and pharmacy' category includes "places like grocery markets, food warehouses, farmers markets, specialty food shops, drug stores, and pharmacies" (Google 2022b). In the 'parks' category, Google condenses "places like local parks, national parks, public beaches, marinas, dog parks, plazas, and public gardens" (Google 2022b). The 'retail and recreation' category combines "places like restaurants, cafes, shopping centers, theme parks, museums, libraries, and movie theaters" (Google 2022b).

According to Google, the mobility data shows "how visits to (or time spent in) categorized places change compared to our baseline days" (Google 2021b). These 7 baseline days (for each day of the week) are the median values from the period Jan 3 till Feb 62020 . We use this information and recalculate the mobility estimates to represent percentage-point changes from our new baseline week (March 23 - 29, 2020). Despite the fact that Google provides no information on the daily absolute mobility of the baseline week, it is possible to fully account for changes of the baseline week. This is described in equation (2) calculating the daily average of mobility measure $y_{k, i}$. Where $x_{k, i}$ is the daily average of mobility measure for each day $i$ and mobility place category $k$ and $\alpha_{m, k}$ is the mobility value of the new baseline day for each place category corresponding to the specific day of the week $m$. Afterwards we calculate the average of the weekly average mobility changes across the place categories and 
match this with the mobility estimates of the GSM and survey based data.

$$
y_{k, i}=\frac{x_{k, i}-\alpha_{m, k}}{1+\alpha_{m, k}}
$$

\section{B) Baseline week}

Our baseline week (March 23-29) contains no major Austrian holidays or (non-pandemic related) school closures. Nevertheless, the choice of the baseline week can have substantial effects on our findings. To study how robust our results are to changes in the baseline week of our estimates, we calculated correlations and coefficients for different baselines. Correlation coefficients between the survey and the GSM estimates are unaffected by changes in the selected reference week. Differences only emerge with regard to Google's Mobility Reports, because this estimate relies on the assumption that every relative change in the mobility categories is equally important for relative changes in the overall mobility estimate. The other estimates calculate the aggregate indices first and then calculate the relative changes. We cannot use this approach for the Google data due to lack of data on absolute mobility. Changes in the reference week, however, only have minor consequences for correlations which vary between 0.864 [if wave 11 is used as the reference week] and 0.944 [wave 3] (for context, baseline wave 1 in main text $=0.936)$. The differences are larger with regard to the slopes of the time trends. The coefficients between Google's Mobility Reports and the survey mobility estimates vary between 0.749 [wave 13] and 1.771 [wave 4]. Coefficients also change when comparing survey mobility estimates and the GSM based estimate (2.542 [wave2] - 1.325 [wave 13]). These results can be explained due to the larger increases in the Google and GSM estimates compared to the survey measure. Thus, basing these estimates on these high values reduces variation over time in the Google and GSM datasets. This also explains why subgroup differences remain largely unaffected. Irrespective of changes in the baseline week, we get larger coefficients for male than for female mobility and for middle aged (30-44 \& 
45-59) compared to younger (15-29) and older (60-74) age cohorts. Coefficients for work are consistently smaller than those for the sum index and coefficients for shopping are consistently larger than those from the main index. The relation remains quite close to 1 for food and medicine (0.873 [wave 7] - 1.176 [wave 1]).

\section{C) Mobility trends by subgroups}

Figure 2 shows the trends in relative mobility by subgroup throughout the pandemic analog to Figure 1 in the main manuscript. Overall gender differences are quite similar when comparing mobile phone and survey data. Both estimate slightly higher relative increases in mobility after the lockdown for women compared to men. While these differences remain quite stable in the survey data, they disappear after summer 2020 in the GSM data. Notably, mobile phone data suggests higher effects of the end of the lockdown in April and May 2020 and at the beginning of 2021. Absolute mobility trends are depicted in Figure 4. These trends

show that, while mobility of women increased more than mobility of men, absolute mobility estimates are always higher for men than for women. Again, the differences in mobility estimates between the two data sources are quite small. The results are slightly different focusing on subgroup differences by age. Younger and older subjects generally show higher relative increases in mobility compared to others (see Figure 2). As suggested in the main text, this might stem from differences in the importance of work-related mobility in these age groups. Because work-related mobility remained important during the lockdown - as not all workers could work from home - the working age population had fewer opportunities to decrease their mobility. This higher mobility level decreases the potential for relative increases after the end of the lockdown, as larger absolute changes in mobility nevertheless appear smaller in the relative mobility estimate. In line with this interpretation, the lockdown in the end of 2020 seems to have decreased these age differences. Again, increases in mobility at the beginning of 2020 and 2021 are more pronounced in the mobile phone data compared to the survey estimates. Figure 4 containing trends of absolute mobility estimates by age-group 
supports this explanation, as those in working age show lower increases in mobility in the first half of 2020 compared to the younger and older age groups. The absolute mobility estimates also indicate that mobility decreases with higher age both in the GSM and in the survey data.

Figure (3) compares survey and Google based relative mobility trends. As noted in the main text, work related mobility is more pronounced in the survey estimate compared to the Google indicator. The difference points in the other direction for mobility related to 'shopping (other)' (=Google's 'retail and recreation' place category). Mobility in the category 'shopping (food and medicine)' is quite similar comparing Google's mobility reports and our survey based mobility estimates. In line with the idea that this mobility category captures essential parts of mobility, the graph indicates lower increases after the lockdown compared to the other mobility categories. Figure 5 focusing on differences in absolute mobility for the GSM and survey estimates shows that these relative differences emerge also due to large differences in the initial raw mobility values. As Google does not provide their absolute mobility estimates, the graph shows the relative changes based on Google's initial baseline. This indicates large relative decreases in the mobility related to 'shopping (other)'. While also low in the survey data ( 0.1 , indicating the average answer was between 'never' and 'on some days'), the survey mobility estimate increased less than the Google estimate. Besides higher initial values at the start of the pandemic in the survey estimate, this could also be because Google's 'retail and recreation' place category covers more mobility categories (such as visits to restaurants and movie theaters) than our corresponding survey measure asking subjects to think of mobility frequency due to 'other procurements'. 


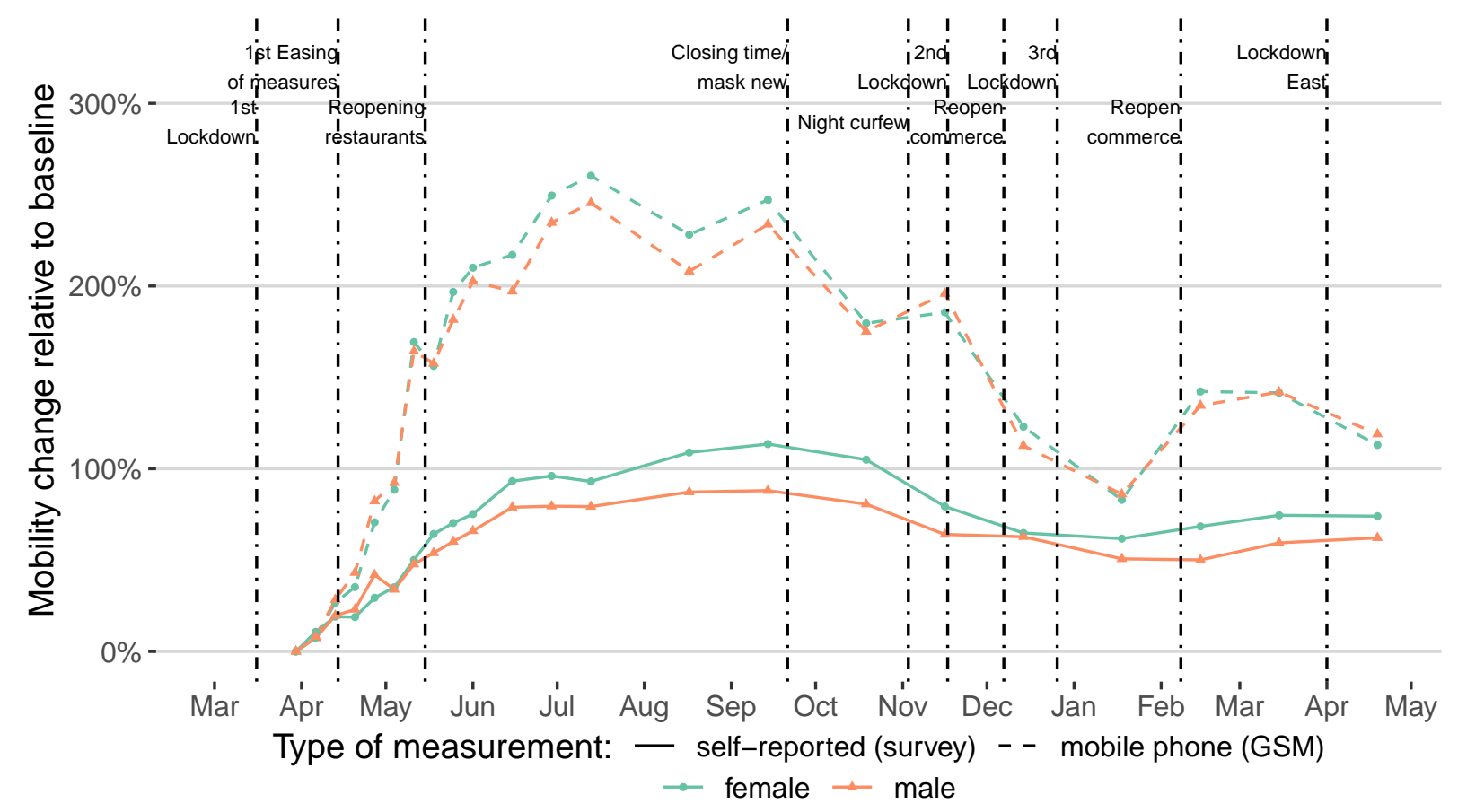

(a) By gender

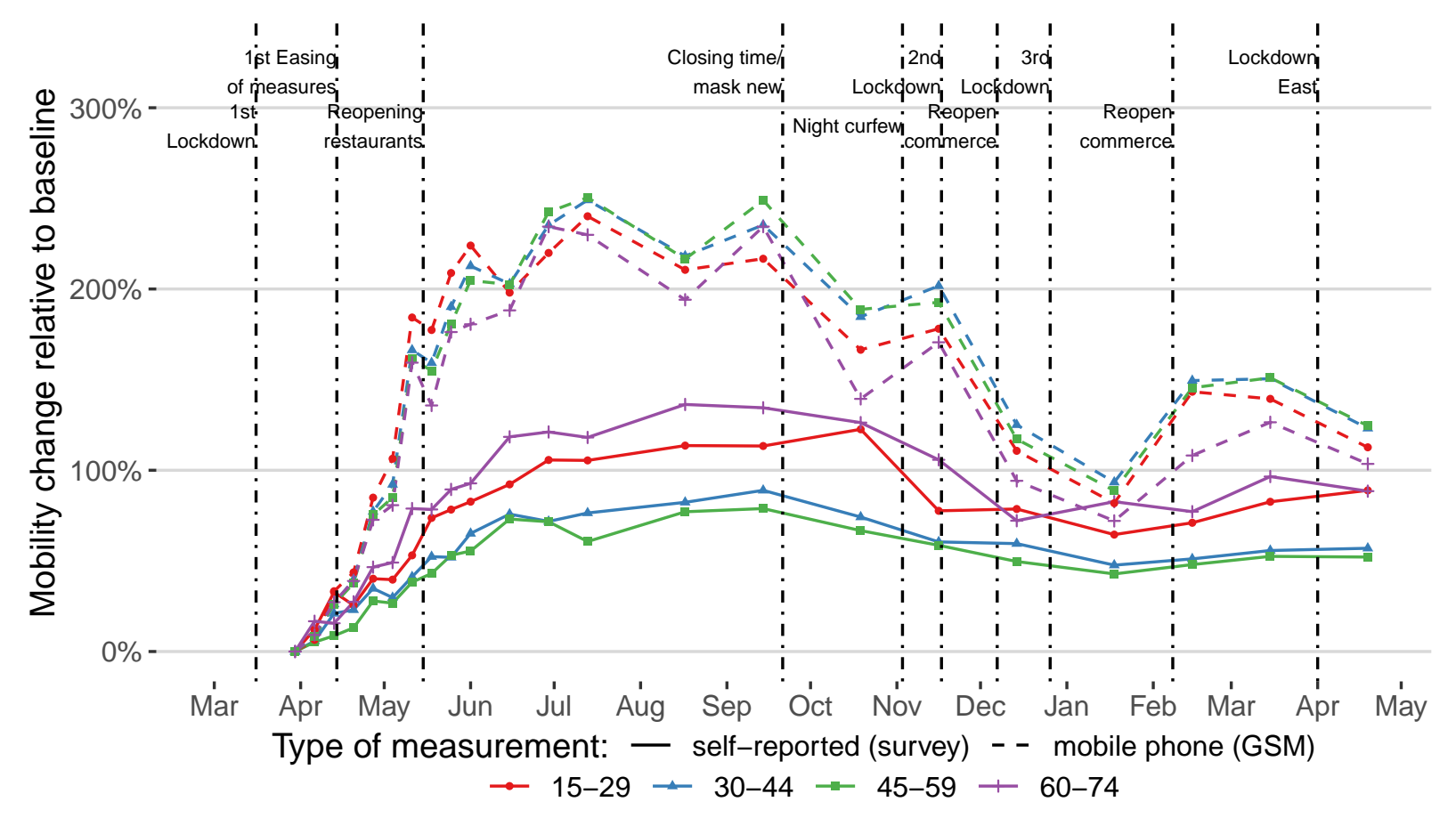

(b) By age group

Figure 2: Trends in relative mobility estimates (average by subgroup) 


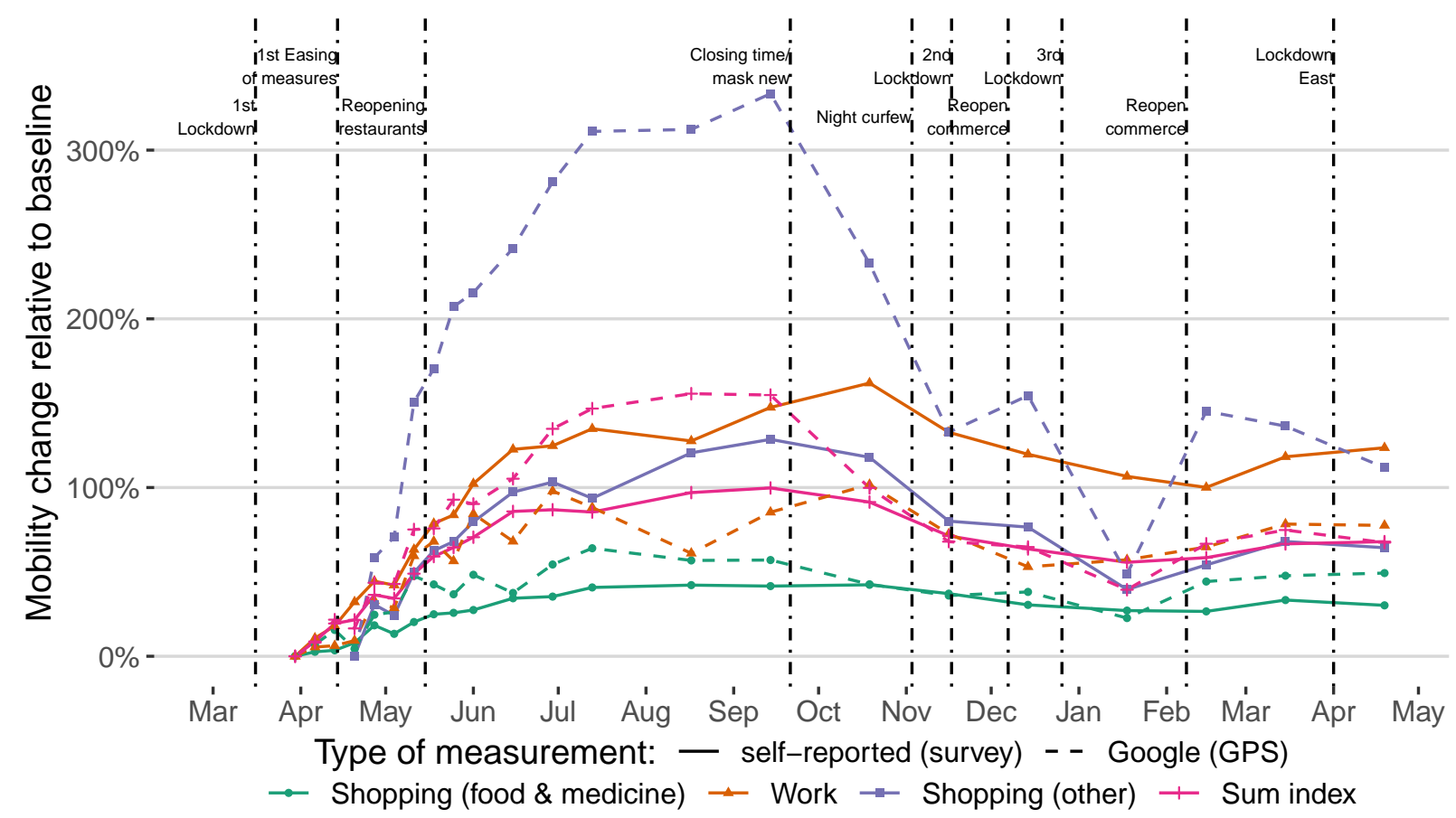

Figure 3: Trends in relative mobility estimates (average by subgroup)
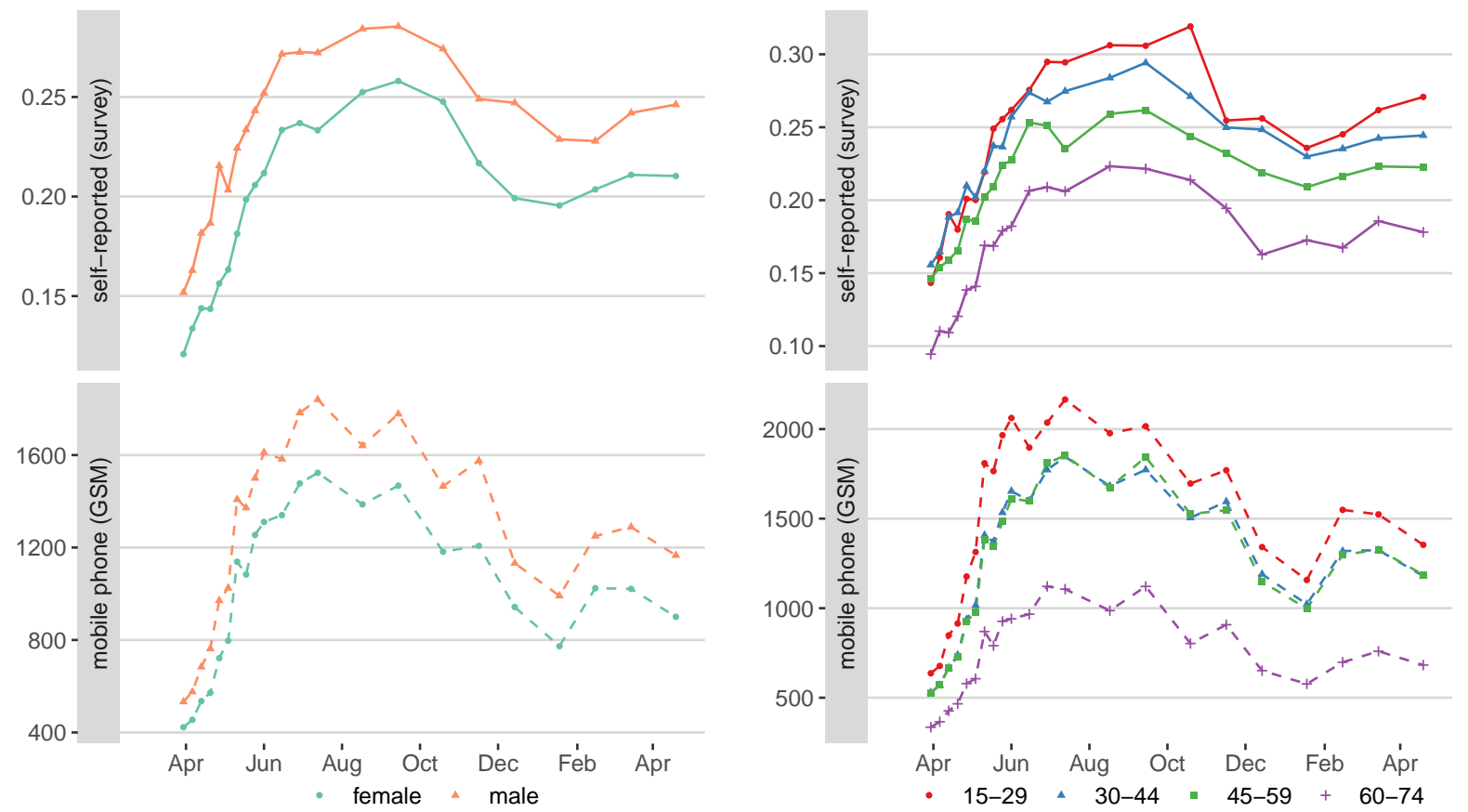

Figure 4: Trends in absolute mobility estimates (average by subgroup) 

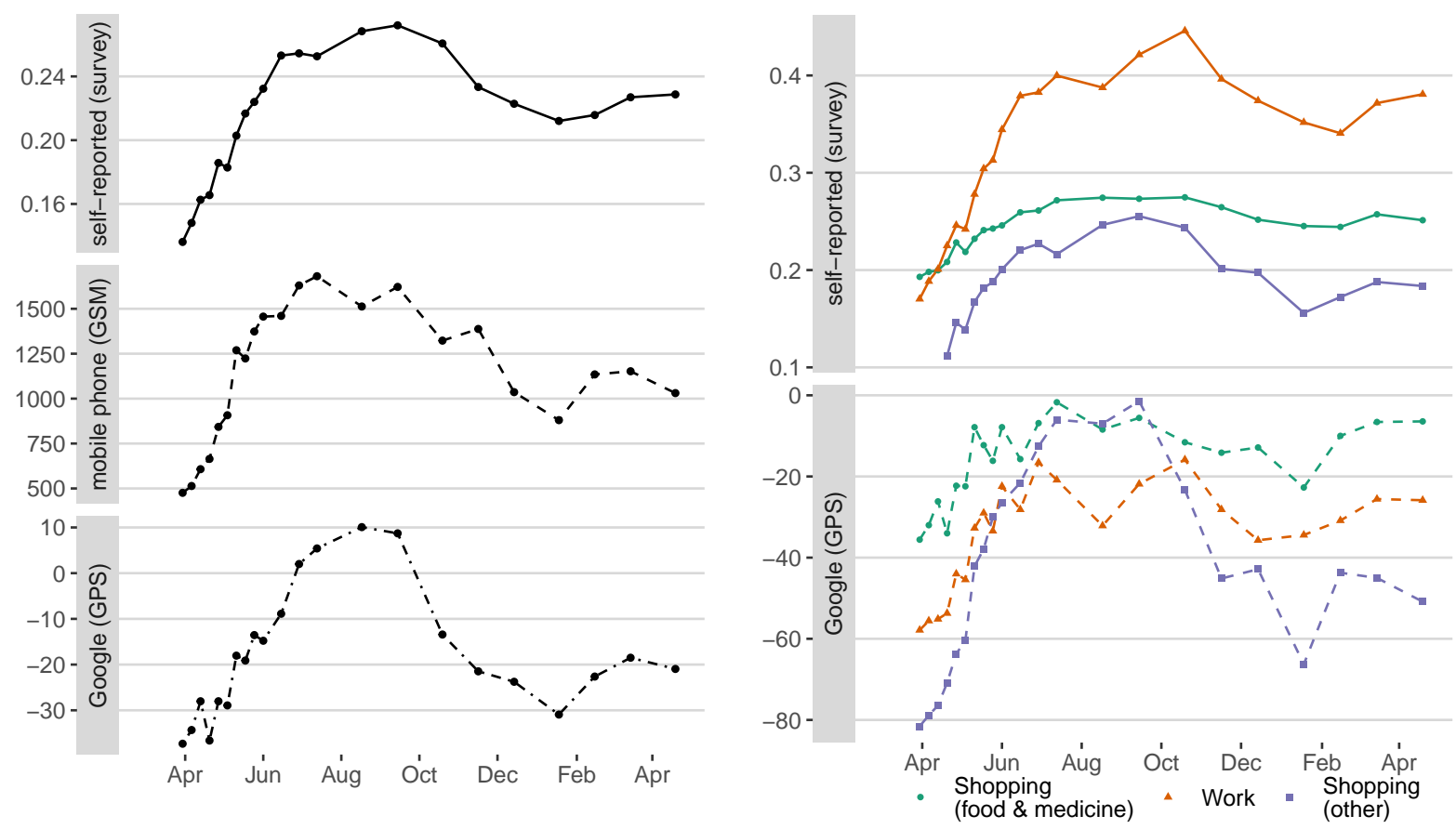

Figure 5: Trends in raw (Google) and absolute (survey) mobility estimates (average by mobility category)

\section{D) Model based comparisons using linear regressions with time}

\section{fixed-effects}

In addition to basic correlation coefficients, we also calculated regressions on aggregated mobility estimates with time fixed effects including dummy variables for the data sources (models 1-4), subgroups (models 3-4), and their interactions (to account for heterogeneous

effects across data sources). The coefficient estimates of these models are presented in Table 2. Model 1 indicates larger variance in mobility over time in the GSM based mobility estimate compared to the survey. In model 2, we test whether this result differs depending on the stringency of governmental measures aimed to limit the spread of the virus. The estimated coefficients show that the differences are becoming smaller if stringency increases. This result suggests that the survey estimates do not suffer from considerable social desirability bias. Model 3 supports our results depicted in Figure 2 in the main manuscript. The coefficient estimates indicate only marginal differences between genders across the GSM and survey 
based mobility estimates. In addition, the relatively reduced mobility changes for middle age categories are less pronounced in the GSM estimate compared to those in the survey data. Similarly, model 4 supports the results displayed in Figure 3 of the main manuscript. Mobility estimates in the category 'shopping (food and medicine)' are smaller in comparison to the overall measure and larger for the mobility categories 'work' and 'shopping (other)'. The regression coefficients of the interactions indicate that variance over time in the category 'work' is smaller in the Google data compared to the survey data. This relationship is flipped in the case of mobility in the category 'shopping (other)'. 
Table 2: Relative weekly mobility: OLS regression estimates

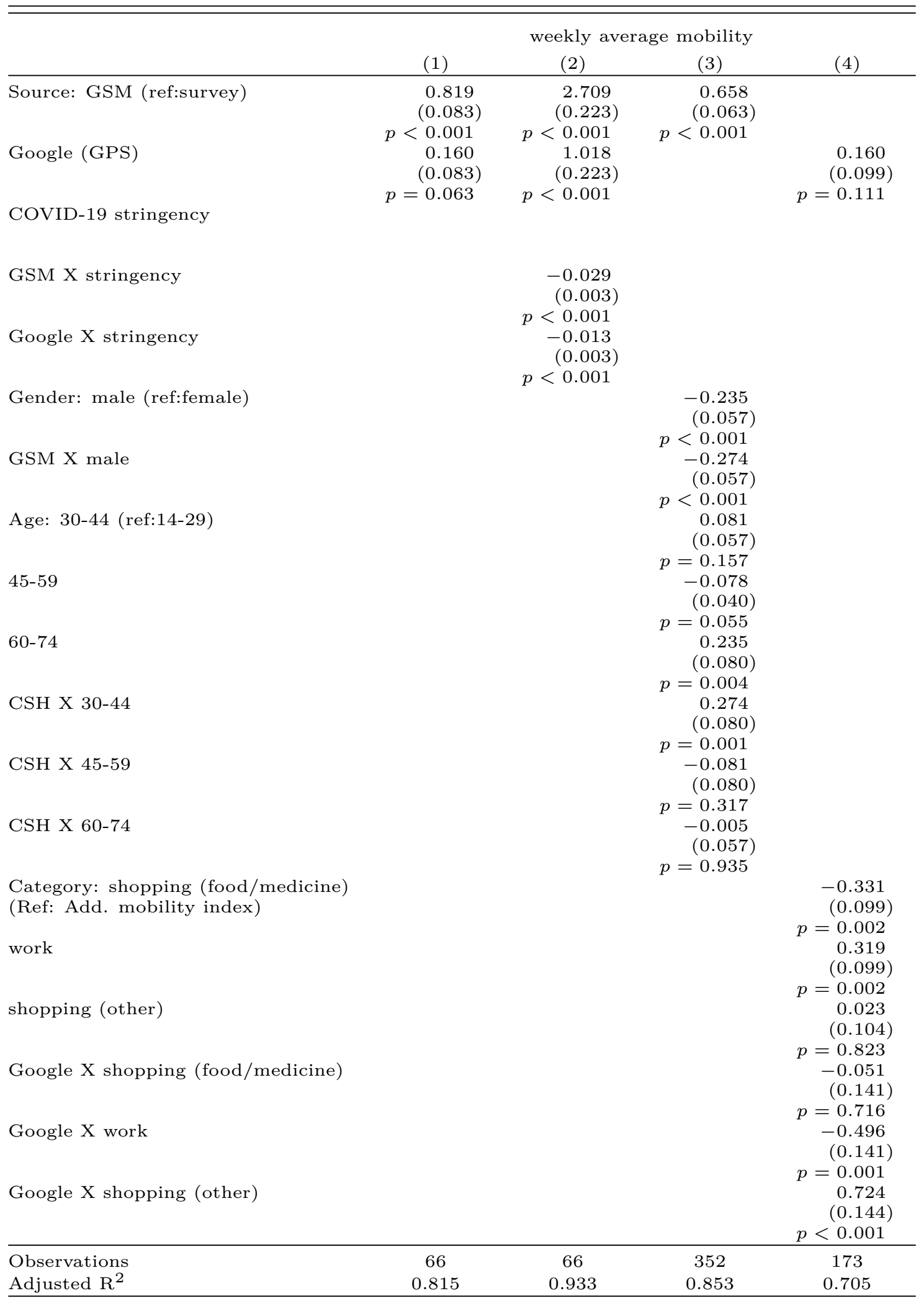

Note: Dependent variable = Relative weekly average mobility by source (model 1-2), by source, gender, and age-group (model 3), by source and mobility category (model 4). Standard errors within parentheses. Regressions include wave fixed effects not shown here. 


\section{E) Results using a single item measure}

To check the robustness of our results, we calculate similar analyses using a second mobility measure that utilizes respondent's self-reported frequency of leaving their home. This variable is only available every second wave. Specifically we compare the weekly absolute shares of people having a ROG of more than 500 meters to the absolute normalized survey wave average of a question asking for respondents' frequency of staying at home (we inverse this variable - question wording can be found in Appendix E). The results reported in Figure 6 and Figure 7 are quite similar to the ones using the additive index. Correlations are high and rather consistent. Also, the differences between genders are consistent across the estimates. Differences between age groups indicate that younger individuals are exceeding the mobility thresholds more frequently, which is particularly visible in the survey measure. The trends in the GSM measure might indicate that this indicator reaches upper bounds with lower age groups. However, also here, the elderly are clearly less mobile.

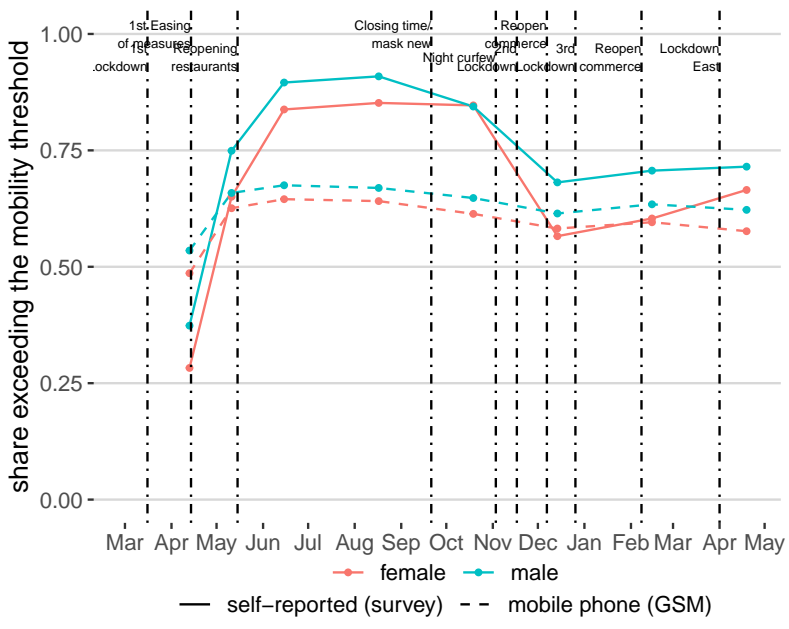

(a) By gender

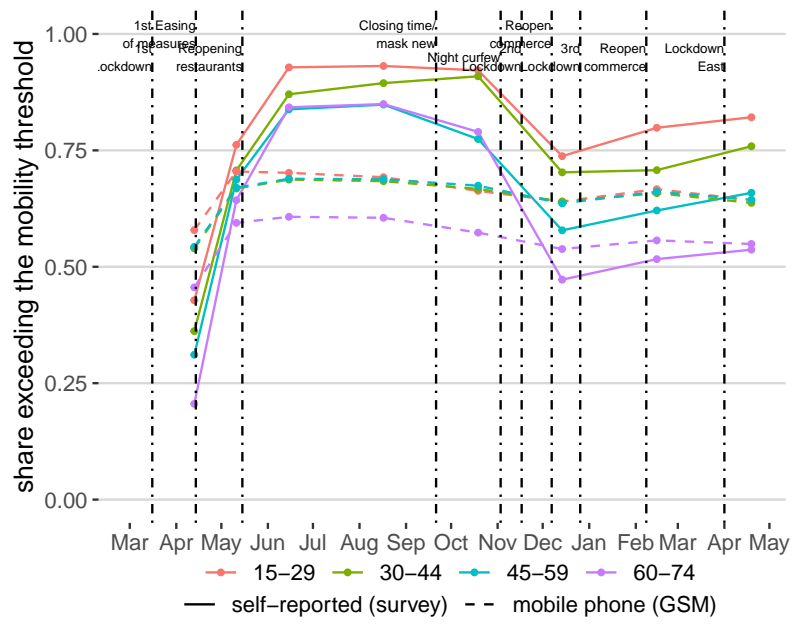

(b) By age group

Figure 6: Trends in mobility estimates (average by subgroup) 


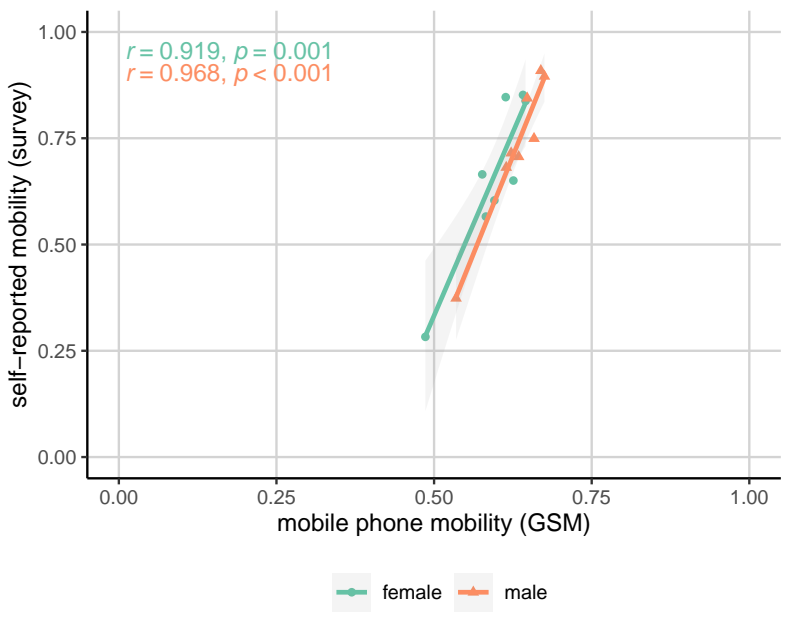

(a) By gender

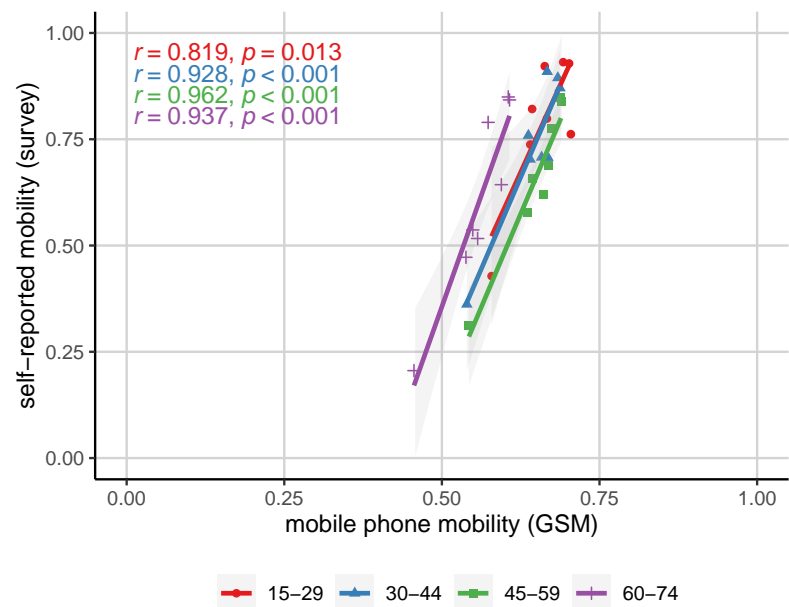

(b) By age group

Figure 7: Correlations of mobility estimates (average by subgroup)

\section{F) Changes between waves: Estimating the effects of the "Lockdown in the East"}

One of the most prominent applications of mobility estimates during the pandemic is to calculate the effectiveness of different governmental measures targeted to reduce mobility and thus limit the spread of the virus. An important measure was the so-called "lockdown", which, in Austria, prominently named the closure of all shops and services except for "essential" branches such as supermarkets or bakeries. While this measure was enacted nationwide most of the time, the infection situation in March 2021 led the regional governments of Burgenland, Lower Austria, and Vienna (all in the east of Austria) to pass a lockdown limited to those specific provinces starting on $1^{\text {st }}$ of April. Burgenland returned to the nationwide rules on $19^{\text {th }}$ of April, while Vienna and Lower Austria prolonged the lockdown until $2^{\text {nd }}$ of May. We use this disparity within Austria for a Difference in Difference (DiD) estimation of the effectiveness of regional lockdowns on reducing mobility by comparing the mobility in Austria's east and west using all three different estimates. If estimates follow the same underlying trends in mobility, we would expect somewhat similar sizes of the overall lockdown effect. Moreover, as possibilities for shopping were most affected by the regional lockdown, 
we would expect higher lockdown effects in this mobility category.

To estimate the DiD analyses we leverage information of respondents' home region in the survey data and utilize the fact that Google provides mobility estimates by region in addition to the estimates by country. Furthermore, the GSM data also contains information about the regional variation of the daily median ROG. In this dataset, the home location is calculated using the nighttime location of the mobile devices.

We assessed the validity of this $\mathrm{DiD}$ estimation strategy by first testing the critical parallel trends assumption. Figure 8 visually confirms that trends in average mobility estimates in all three datasets between March 2020 and March 2021 (i.e., pre-treatment) were rather similar in the treatment group (Burgenland, Lower Austria, and Vienna) and control group (Carinthia, Upper Austria, Salzburg, Styria, Tyrol, and Vorarlberg). Figure 9 shows that this also holds if we focus on mobility in the category shopping (other) in those data sources with distinguishable mobility categories.

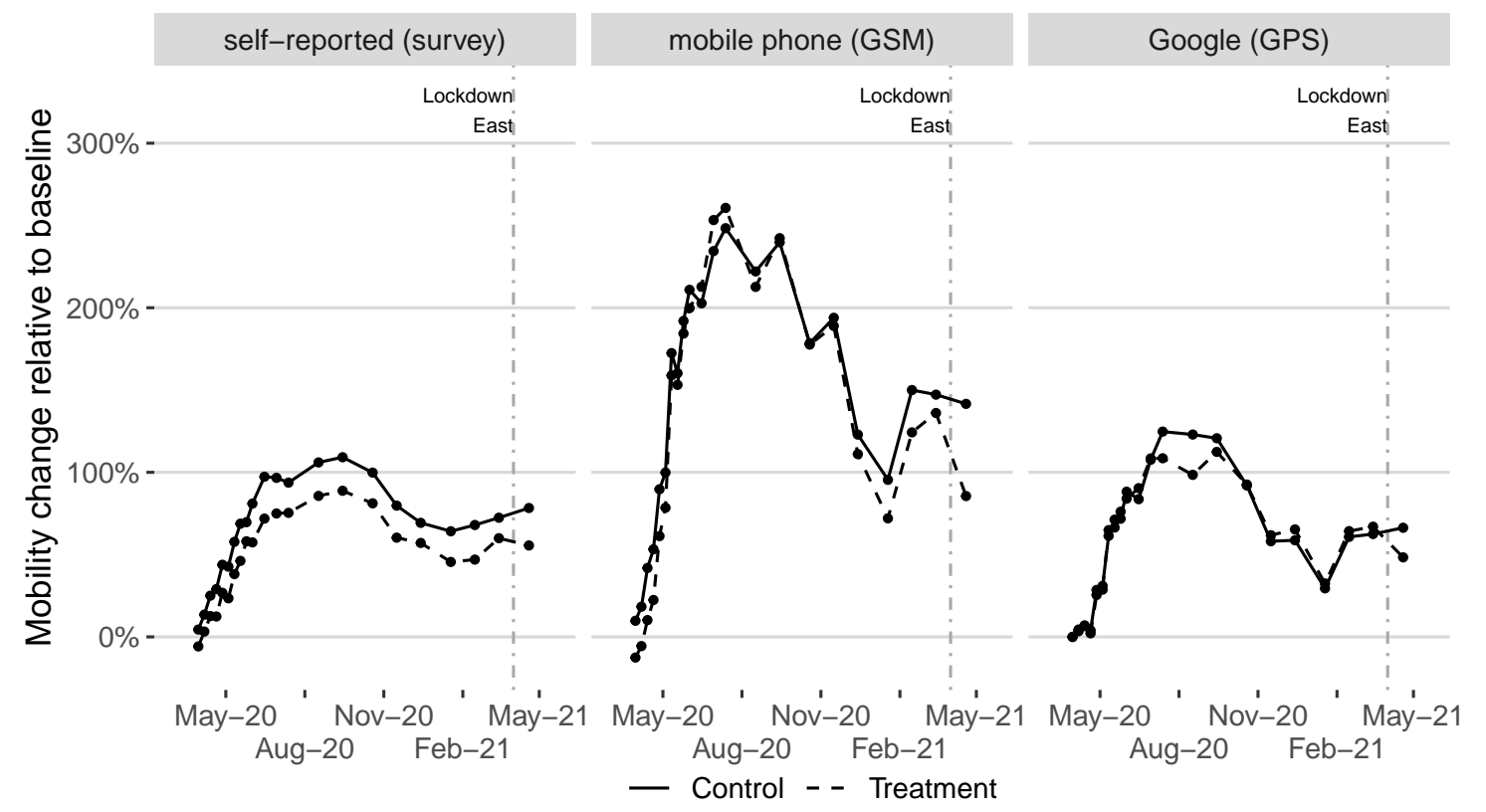

Figure 8: Mobility in treatment and control group over time (all)

Figure 8 shows an increase in mobility between March 2021 and April 2021 in the control 


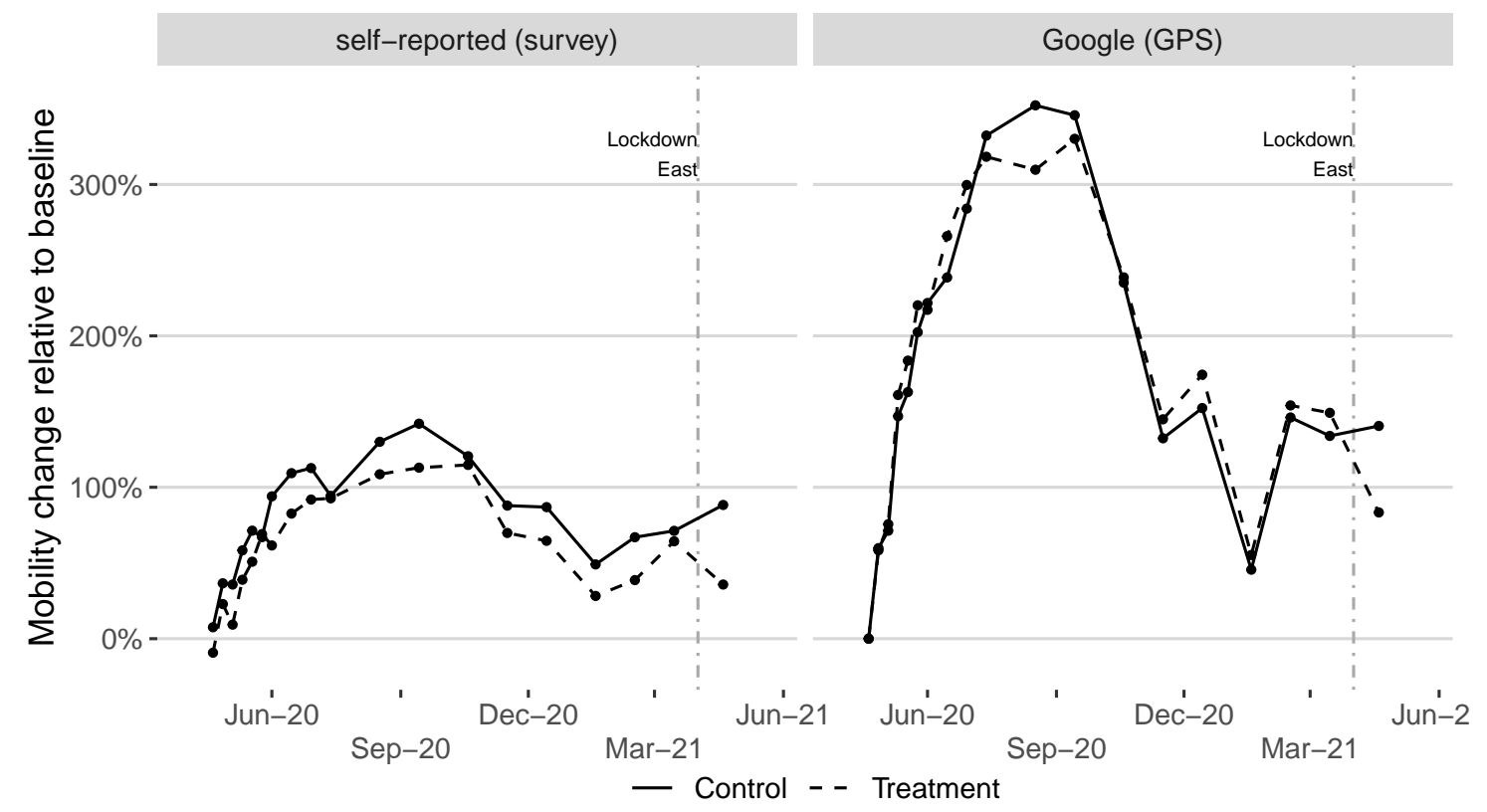

Figure 9: Mobility in treatment and control group over time (shopping -other)

group and a clear decrease in the treatment group within this time frame. Our DiD approach enabled us to estimate the causal impact of this lockdown treatment (Table 1). The constants describe the average mobility in the control group before the treatment. The coefficients suggest higher mobility changes from the baseline week in the control group ahead of the treatment in the GSM and the GPS (shopping) estimates compared to the survey estimate. We now focus on the interaction effect of treatment and time, representing our main estimates for the lockdown effect. Regression coefficients suggest that the main mobility measures utilizing Google's mobility reports (model 1) changed by 22.5 percentage points due to the lockdown. Utilizing mobility measures only for places related to retail and recreation (model 2 ), the coefficient increases to 72.4 percentage points. This is also captured visually in the more pronounced variation between March and April of 2021 in Figure 9.

Structurally similar results are obtained when using survey data: model 4 suggests a reduction in mobility of 14.1 percentage points in the East due to the lockdown (not statistically significant at the .05 level). This effect strongly increases in model 5 , focusing on the single mobility measure indicating how often people went shopping (47.8 percentage points, $\mathrm{p}=$ 
0.002). The GSM data (model 3) shows the most pronounced general effect of the Lockdown in Austria's east, indicating a reduction in mobility by 44.9 percentage points. Again, this shows the higher variation of this measure over time. Generally, however, all measures capture a reduction in mobility in Austria's East due to the Lockdown.

In addition, we note that differences between estimates could also stem from different definitions of an individual's home region. While cross-regional commuting traffic and tourism within Austria contribute to the mobility of an individual's usual home-region in the GSM and possibly also in the survey data, this is likely to be different for the mobility estimate reported by Google.

Table 3: Diff in Diff estimates of Lockdown East

\begin{tabular}{|c|c|c|c|c|c|}
\hline & \multicolumn{5}{|c|}{ Mobility estimate: } \\
\hline & GPS (all) & $\begin{array}{l}\text { GPS (shopping) } \\
\text { Google }\end{array}$ & $\begin{array}{l}\text { GSM } \\
\text { CSH }\end{array}$ & Self-reported (all) & $\begin{array}{l}\text { Self-reported (shopping) } \\
\text { ACPP }\end{array}$ \\
\hline & $(1)$ & $(2)$ & $(3)$ & $(4)$ & $(5)$ \\
\hline Treatment & 0.045 & 0.153 & -0.111 & $\begin{array}{c}-0.074 \\
(0.062) \\
p=0.234\end{array}$ & $\begin{array}{r}0.060 \\
(0.109) \\
p=0.586\end{array}$ \\
\hline Time & 0.038 & 0.066 & -0.056 & $\begin{aligned} & 0.035 \\
&(0.059) \\
& p= 0.553\end{aligned}$ & $\begin{array}{r}0.164 \\
(0.105) \\
p=0.118\end{array}$ \\
\hline Treatment X Time & -0.225 & -0.724 & -0.449 & $\begin{array}{r}p-0.141 \\
(0.089) \\
p=0.116\end{array}$ & $\begin{aligned} & 0.478 \\
& (0.158) \\
p= & 0.003\end{aligned}$ \\
\hline Constant & 0.625 & 1.339 & 1.472 & $\begin{aligned} & 0.740 \\
& (0.041) \\
p< & 0.001\end{aligned}$ & $\begin{array}{r}0.709 \\
(0.073) \\
p<0.001\end{array}$ \\
\hline Observations & 4 & 4 & 4 & 2,494 & 2,494 \\
\hline
\end{tabular}

Note: As we only observe aggregate changes within the Google and GSM measures we cannot calculate estimates on the statistical variance of these effects with this data. Standard errors within parentheses. 


\section{G) Question wordings}

1. Frequency respondents leave their home by reason of mobility:

Have you left your home last week for the following reasons?
a) Work
b) Physical activity
c) Meeting friends or family
d) To buy medicine, receive medical care or to care for/help others
e) To buy food
f) To walk the pet
g) Due to boredom or to enjoy my freedom
h) Other procurements [always in penultimate position]
i) Other [always in last position]

Matrix labels:

- never

- on some days

- several times a week

- almost every day

- daily

- don’t know

- no answer

2. Frequency respondents stay at home:

In the following, think about your personal behavior during the last week. Please indicate how often you have behaved as follows: a) You stay at home, except for necessities.

Matrix labels:

- almost always

- mostly

- sometimes

- rarely

- almost never

- don't know

- no answer

\section{Literature}

ACPP (Austrian Corona Panel Project). 2021. "Austrian Corona Panel Data: Method Report." https://viecer.univie.ac.at/coronapanel/austrian-corona-panel-data/method-report/. 
Fox, John and Sanford Weisberg Nyrup. 2019. "An R Companion to Applied Regression", Third Edition, London: Sage.

Gauvin, Laetitia, Michele Tizzoni, Simone Piaggesi, Andrew Young, Natalia Adler, Stefaan Verhulst, Leo Ferres, and Ciro Cattuto 2020. "Gender Gaps in Urban Mobility." Humanities and Social Sciences Communications 7 (1): 1-13. https://doi.org/10.1057/s41599-020-0500-x.

Google. 2021a. "COVID-19 Community Mobility Report." COVID-19 Community Mobility Report. https://www.google.com/covid19/mobility?hl=en.

_ 2021b. "Overview - Community Mobility Reports Help." https://support.google.com /covid19-mobility/answer/9824897?hl=en\&ref_topic=9822927\#zippy=.

. 2022a. "How Google Uses Location Information." https://policies.google.com/techno logies/location-data?hl=en.

—. 2022b. "Mobility Data Report CSV Documentation." https://www.google.com/covid 19/mobility/data_documentation.html?hl=en.

Heiler, Georg, Tobias Reisch, Jan Hurt, Mohammad Forghani, Aida Omani, Allan Hanbury, and Farid Karimipour. 2020. "Country-Wide Mobility Changes Observed Using Mobile Phone Data During COVID-19 Pandemic." arXiv:2008.10064. http://arxiv.org/abs/2008.10064.

Pappalardo, Luca, Filippo Simini, Salvatore Rinzivillo, Dino Pedreschi, Fosca Giannotti, and Albert-László Barabási. 2015. "Returners and Explorers Dichotomy in Human Mobility." Nature Communications 6 (1): 8166. https://doi.org/10.1038/ncomms9166.

Pepe, Emanuele, Paolo Bajardi, Laetitia Gauvin, Filippo Privitera, Brennan Lake, Ciro Cattuto, and Michele Tizzoni. 2020. "COVID-19 Outbreak Response, a Dataset to Assess Mobility Changes in Italy Following National Lockdown." Scientific Data 7 (1): 230. https: //doi.org/10.1038/s41597-020-00575-2.

Reisch, Tobias, Georg Heiler, Jan Hurt, Peter Klimek, Allan Hanbury, and Stefan Thurner 2020. "Behavioral Gender Differences Are Reinforced during the COVID-19 Crisis." arXiv:2010.10470. https://arxiv.org/abs/2010.10470.

Statistik Austria 2019. "Austrian Micro Census - Labour Force Survey Yearly Data." https: //data.statistik.gv.at/web/meta.jsp?dataset=OGD_ake001j_AKEJ_1

Statistik Austria 2021. "IKT-Einsatz in Haushalten 2021." http://www.statistik.at/web _de/statistiken/energie_umwelt_innovation_mobilitaet/informationsgesellschaft/ikteinsatz_in_haushalten/index.html

TU Wien, DWH, and CSH consortium. 2021. "Synthetic population COVID model." https://syd19.netlify.app/.

Vehovar, Vasja, Vera Toepoel, and Stephanie Steinmetz. 2016. "Non-Probability Sampling". 
In: The SAGE Handbook of Survey Methodology London: Sage, 329-45. https://dx.doi.org/1 0.4135/9781473957893.n22.

Wu, Laiyun, Samiul Hasan, Younshik Chung, and Jee E. Kang. 2021. "Understanding the Heterogeneity of Human Mobility Patterns: User Characteristics and Modal Preferences." Sustainability 13 (24): 13921. https://doi.org/10.3390/su132413921. 